\title{
Dickkopf1 destabilizes atherosclerotic plaques and promotes plaque formation by inducing apoptosis of endothelial cells through activation of ER stress
}

\author{
Mingxue $\mathrm{Di}^{1,2,4}$, Lin Wang ${ }^{1,2,3,4}$, Mengmeng $\mathrm{Li}^{1,2}$, Yu Zhang ${ }^{1,2}$, Xinxin Liu ${ }^{1,2}$, Renya Zeng ${ }^{1,2}$, Han Wang ${ }^{1,2}$, Yifei Chen ${ }^{1,2}$, Weijia Chen ${ }^{1,2}$, \\ Yun Zhang ${ }^{1,2}$ and Mei Zhang ${ }^{*, 1,2}$
}

Several clinical studies reported that Dickkopf1 (DKK1) plasma levels are correlated with atherosclerosis. However, the impact of DKK1 on the formation and vulnerability of atherosclerotic plaques remains elusive. This study investigated DKK1's effects on enlargement and destabilization of plaques by targeting endothelial cells and assessing the possible cellular mechanisms involved. The effects of DKK1 on atherogenesis and plaque stability were evaluated in ApoE $-I-$ mice using lentivirus injections to knockdown and knock-in the DKK1 gene. The presence of DKK1 resulted in enlarged and destabilized atherosclerotic lesions and increased apoptosis, while silencing of DKK1 alleviated plaque formation and vulnerability in the whole progression of atherosclerosis. DKK1 expression was upregulated in response to ox-LDL treatment in a time- and concentration-dependent manner on human umbilical vein endothelial cell (HUVEC). The interference of DKK1 reversed ox-LDL-induced apoptosis in HUVECs. The mechanism underlying this effect was DKK1's activation of the JNK signal transduction pathway and inhibition of canonical Wnt signaling, following by activation of the IRE1 $\alpha$ and eif2 $\alpha /$ CHOP pathways. In conclusion, DKK1 promotes plaque formation and vulnerability partly by inducing apoptosis in endothelial cells, which partly through inducing the JNK-endoplasmic reticulum stress pathway and inhibiting canonical Wnt signaling.

Cell Death and Disease (2017) 8, e2917; doi:10.1038/cddis.2017.277; published online 13 July 2017

Recent studies have found that acute coronary syndrome (ACS) is associated with both the sudden rupture of atherosclerotic plaques and the rapid development of these plaques. While atherogenesis remains incompletely understood, studies of atherogenesis pathology suggest that multiple cellular malfunctions, including endothelial cell (EC) dysfunction and vascular integrity disruption, are involved, as well as increases in inflammatory cell numbers, the production of cytokines, the proliferation and migration of vascular smooth muscle cells (VSMCs), the activation of monocytes and macrophages, and neovascularization. Pathological biomechanical and haemodynamic changes (e.g., oxidative damage, shear stress) result in these events, which are closely correlated with plaque stability. ${ }^{1}$ EC dysfunction has been observed in atherosclerotic lesions in both humans and animals and eventually leads to apoptosis and the development of atherosclerosis. ${ }^{2,3}$ Endothelial dysfunction leads to proinflammatory activation, generates autocrine, and paracrine signaling loops, and influences other type of cells that are involved in atherogenesis. ${ }^{4}$ EC apoptosis occurs throughout the early stages of atherosclerosis and plays important roles in plaque regression and plaque instability, 5,6 which are caused by various factors, particularly by oxidized low-density lipoprotein (ox-LDL). ${ }^{1,7}$ Thus, inhibition of EC apoptosis may be a useful therapeutic strategy for ameliorating plaque instability.

Dickkopf1 (DKK1), a secretory glycoprotein, can block the Wnt pathway by competitively binding to receptors (e.g., LRP5/6, Kremen) on the cell membrane. ${ }^{8}$ Ueland et al. found that DKK1 expression was stronger in von Willebrand factor (vWF)-positive ECs and in CD68-positive macrophages than in other areas of plaques. $^{9}$ They also found that DKK1 participated in platelet-induced EC activation, indicating that DKK1 promotes inflammation in atherosclerotic plaques and is an atherogenic factor. ${ }^{9}$ In a previous clinical study of patients with ACS, we found that DKK1 plasma levels were not only correlated with disease severity but also served as a prognostic predictor. Thus, DKK1 concentration may reflect the severity and stability of coronary atherosclerosis. ${ }^{10}$ Several studies have indicated that DKK1 plays an important role in atherosclerosis; however, the underlying mechanisms have yet to be elucidated. Furthermore, the knockout mouse is not an ideal model for DKK1 research in disease, because the homozygous DKK1 $(-/-)$ mutation is lethal. ${ }^{11}$ Therefore, in this investigation, we used a lentivirus to overexpress or silence DKK1 in ApoE - / - mice.

A previous study found a strong association between endoplasmic reticulum stress (ERS) markers, such as CCAAT/

\footnotetext{
${ }^{1}$ Key Laboratory of Cardiovascular Remodeling and Function Research, Chinese Ministry of Education and Chinese Ministry of Health, Qilu Hospital, Shandong University, Jinan 250012, China; ${ }^{2}$ The State and Shandong Province Joint Key Laboratory of Translational Cardiovascular Medicine, Qilu Hospital, Shandong University, Shandong 250012, China and ${ }^{3}$ Department of Gerontology, The Second Hosipital of Shandong University, Jinan 250012, China

*Corresponding author: M Zhang, Key Laboratory of Cardiovascular Remodeling and Function Research Department of Cardiology, Qilu Hospital, Shandong University, No.107, Wen Hua Xi Road, Jinan, Shandong 250012, China. Tel: +86 531 82169139; Fax: +86 531 86169356; E-mail: daixh@ vip.sina.com

${ }^{4}$ The authors contributed equally to this work.

Received 30.11.16; revised 10.4.17; accepted 13.4.17; Edited by A Oberst
} 
enhancer-binding protein-homologous protein (CHOP) and glucose-regulated protein 78 (GRP78), and the presence of atherosclerotic plaques in human coronary artery lesions, suggesting that ERS is involved in the development of plaque instability in humans. ${ }^{12}$ Disrupting the secretion of Wnt5a, a Wnt pathway agonist, has been shown to induce ERS in mammalian cells, indicating that a correlation exists between Wnt secretion and ERS. ${ }^{13}$ DKK1 is an important regulator of the Wnt pathway, ${ }^{8}$ yet, its role in ERS-associated apoptosis in atherosclerosis remains unclear.

On the basis of these findings, we hypothesized that DKK1 promotes plaque formation and instability in part by stimulating EC apoptosis. To accomplish this, we investigated the effect of modulated DKK1 expression on atherosclerosis plaques in ApoE - / - mice and EC apoptosis; and explored the underlying mechanisms in endothelial cells using human umbilical vein endothelial cells (HUVECs).

\section{Results}

DKK1 influenced the formation and vulnerability of aortic plaques and caused vascular endothelium dysfunction in ApoE - / - mice. Intense GFP staining was observed in aortic plaques and carotid artery plaques (Figure 1b). The results of Western blotting to reveal aorta-containing proteins, immunohistochemistry and analysis of plasma DKK1 further demonstrated that DKK1 protein expression was significantly lower in the shDKK1 group and higher in the DKK1 group than in the NS and GFP groups (Figures 1c-f), which established that overexpression and silencing vectors were effective.

Endomucin is a marker for endothelial cells, ${ }^{14}$ and MOMA-2 is a marker for monocyte-macrophage. ${ }^{15}$ Similar to the findings of a previous study, ${ }^{9}$ atherosclerotic lesions in the aortic plaques in the ApoE - / - mice showed strong DKK1 expression in all endomucin-positive ECs and in small regions containing MOMA-2-positive macrophages (Figure 1g). Colocalization immunofluorescence staining further confirmed that the majority of DKK1 was expressed in ECs other than smooth muscle cells and macrophages.

Vascular endothelium dysfunction plays an important role in atherosclerosis. When the mice were fed atherogenic chow for 4 weeks, the shDKK1 group showed recuperative acetylcholine-induced endothelium-dependent relaxation in the thoracic aorta ring after pre-contraction with norepinephrine, whereas the DKK1 group showed damaged endothelial relaxation (Supplementary Figure 1).

In mice fed atherogenic chow for 12 weeks, both the en face lesion areas and the cross-section lesion areas measured in the aortic root were decreased in the shDKK1 group and increased in the DKK1 group compared with the areas in the NS and GFP groups (Figures $2 \mathrm{a}-\mathrm{c}$ ).

In both aortic (Figure 2d) and carotid (Figure 2h) plaques, the relative content of VSMCs and collagen fibers were lower in the DKK1 group but higher in the shDKK1 group than in the NS GFP groups. Conversely, the relative contents of macrophages and lipids were higher in the DKK1 group but lower in the shDKK1 group than in the NS and the GFP groups. Accordingly, the plaque vulnerability index was higher in the DKK1 group but lower in the shDKK1 group. Moreover, fibrotic cap thickness was substantially lower in the DKK1 group and higher in the shDKK1 group than in the NS and the GFP groups (Figures $2 \mathrm{~d}$ and $\mathrm{g}$ ). Although plaques were completely filled in most carotid arteries, except in the shDKK1 group, the carotid plaques were shown the similar trend (Figures $2 \mathrm{~h}-\mathrm{j}$ ). Moreover, vascular outward remodeling was more significant in the DKK1 group than in the other groups (Figure 2k). In mice fed atherogenic chow for 4 or 8 weeks, the shDKK1 group displayed substantially increased fibrous caps of plaque, while the DKK1 group showed increased plaque size and vulnerability (Supplementary Figure 2). DKK1 can aggravate plaque vulnerability not only during the short-term feeding of atherogenic diet, but also in the long-term atherogenic diet. These results reveal that DKK1 augments plaque formation and instability in both aortic and carotid plaques and that silencing DKK1 attenuates these effects.

DKK1-induced inflammatory factors and apoptosis in aortic atherosclerotic plaques in ApoE-I- mice. Several studies showed that inflammation and apoptosis contribute to the instability of atherosclerotic plaques. ${ }^{1}$ The content of inflammatory factors (e.g., IL-6, IL-1 1 , MCP-1, and TNF-a) was lower in the shDKK1 group and higher in the DKK1 group $(P<0.05$, Figure $3 a)$.

Expression of the anti-apoptotic factor Bcl-2 was significantly upregulated in the shDKK1 group, while expression of the pro-apoptotic factor Bax and cleaved caspase-3 was significantly downregulated compared to the NS and GFP groups $(P<0.05$, Figure $3 \mathrm{~b})$. The opposite results were found in the DKK1 group $(P<0.05$, Figure $3 \mathrm{~b})$.

The percentage of TUNEL $(+)$ cells was lower in the shDKK1 group and higher in the DKK1 group than in the NS and GFP groups $(P<0.05$, Figures $3 c$ and $d)$. The percentage of TUNEL $(+)$ endomucin $(+)$ cells showed a similar trend $(P<0.05$, Figure $3 e)$. Accordingly, the results from immunofluorescence analysis and TUNEL assay co-localization revealed that DKK1 promoted apoptosis in atherosclerotic plaques, mostly due to its effects on ECs.

DKK1 expression was upregulated following ox-LDL treatment in a time- and concentration-dependent manner, and interference of DKK1 inhibited ox-LDL-induced apoptosis in HUVECs. Oxidized low-density lipoprotein (ox-LDL) may play a pre-eminent function in atherosclerotic lesion formation. ${ }^{16}$ So in vitro, we used ox-LDL to mimic the stimulation to the endothelial cells during atherosclerosis. HUVECs treated with ox-LDL $(150 \mu \mathrm{g} / \mathrm{ml})$ for various lengths of time $(0 \mathrm{~h}, 0.5 \mathrm{~h}, 1 \mathrm{~h}, 3 \mathrm{~h}, 6 \mathrm{~h}$, or $12 \mathrm{~h})$ exhibited time-dependent increases in DKK1 protein and mRNA levels as well as in DKK1 levels in the culture supernatant. DKK1 expression gradually increased at the mRNA level starting from $1 \mathrm{~h}$, at the protein level starting from $3 \mathrm{~h}$, and in the culture supernatant from $6 \mathrm{~h} \quad(P<0.05$, Figures $4 \mathrm{a}-\mathrm{c})$. HUVECs treated for $6 \mathrm{~h}$ with various concentrations of ox-LDL $(0,25,50,100,150$, or $200 \mu \mathrm{g} / \mathrm{ml})$ exhibited concentration-dependent increases in DKK1 mRNA and protein expression and in DKK1 levels in the culture supernatant. DKK1 expression was consistently significantly higher in cells 
a
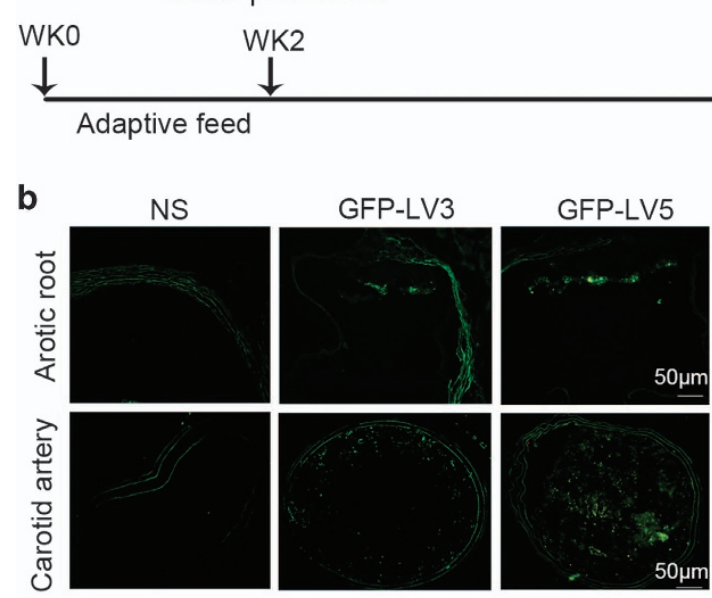

C
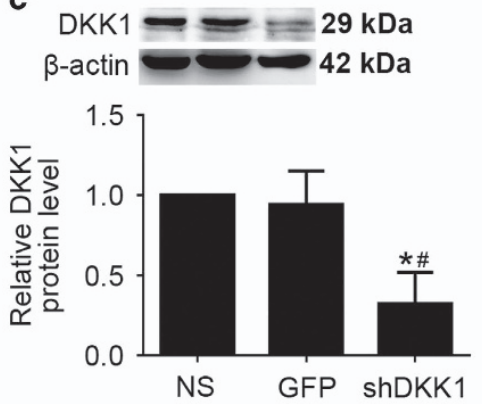

Lentivirus transfection GFP detection Mice sacrificed

$\begin{array}{ccc}\text { WK10 } & \text { WK12 } & \text { WK14 } \\ \downarrow & \downarrow & \downarrow\end{array}$
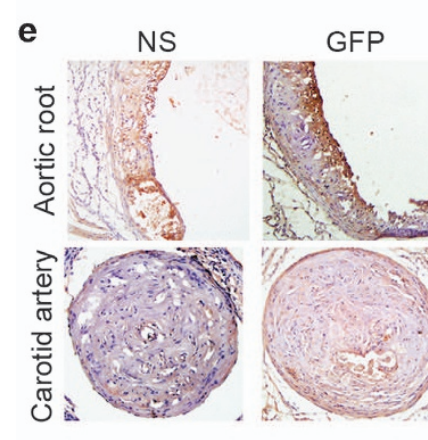

f

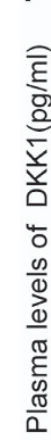

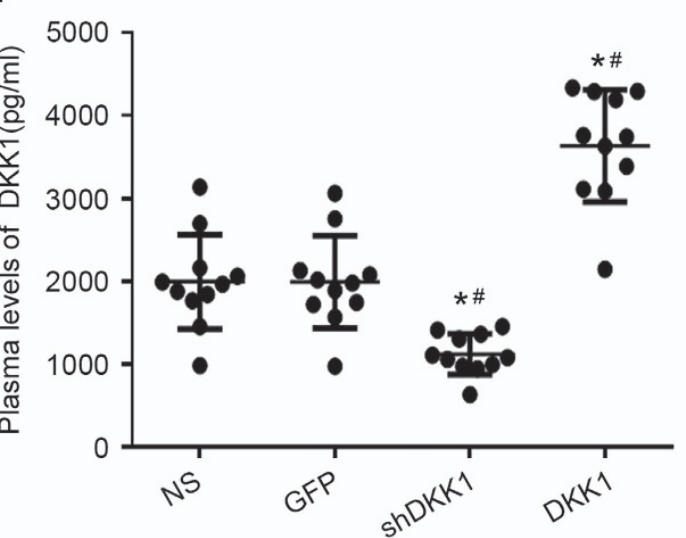

g
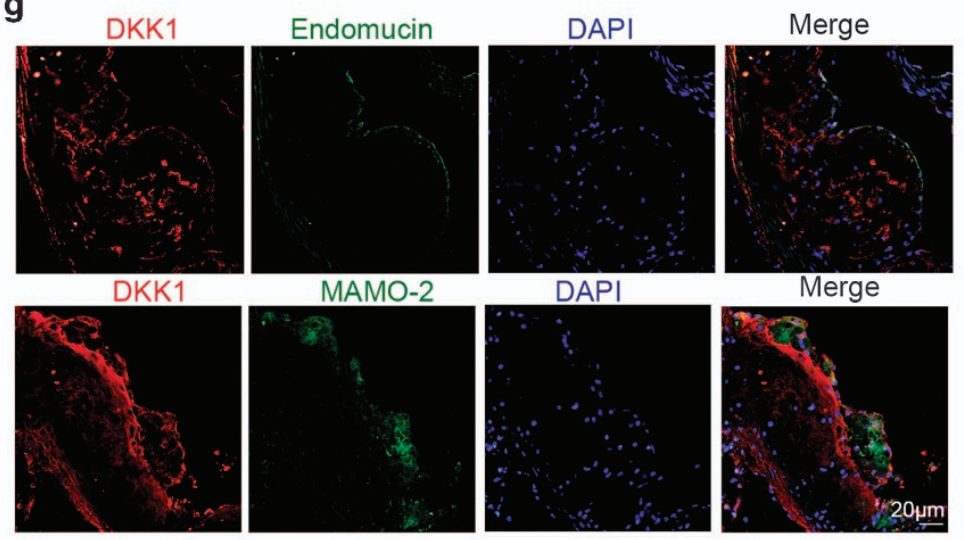

Figure 1 Efficiency of lentivirus transfection in ApoE - / - mice. (a) Flow charts showing the experimental protocol used in the in vivo studies. (b) Fluorescence images of the aortic root and carotid plaques two weeks after lentivirus transfection in the lenti-GFP group. (c,d) DKK1 protein expression in the aortic root in four groups of mice as determined by Western blotting. $n=6$. (e) Areas stained positive for DKK1 in four groups of mice as determined by immunohistochemical staining in the aortic root and carotid plaques. $n=6$. (f) Plasma levels of DKK1 in four groups of mice. $(n=11)$. (g) Co-localization of DKK1 (red) and endomucin (green) or MOMA-2 (green) expression in aortic plaques in the NS group. DAPI (blue) indicates nuclei. Data are shown as the mean \pm S.D., ${ }^{*} P<0.05$ versus NS; ${ }^{\#} P<0.05$ versus GFP

treated with 150 and $200 \mu \mathrm{g} / \mathrm{ml}$ ox-LDL than in cells treated with $0 \mu \mathrm{g} / \mathrm{ml}(P<0.05$, Figures $4 \mathrm{~d}-\mathrm{f})$. These results indicate that DKK1 expression increases in HUVECs in response to treatment with ox-LDL. Based on these results, we chose to treat cells with $150 \mu \mathrm{g} / \mathrm{ml}$ ox-LDL for $6 \mathrm{~h}$ to investigate how DKK1 affects EC function.

We first examined the effect of DKK1 on apoptosis in HUVECs treated with ox-LDL. To accomplish this, cells were transfected with either negative control siRNA (NC) or DKK1 siRNA. The DKK1 siRNA significantly reversed the effects of ox-LDL on the expression of cleaved caspase-3 and the protein ratio of $\mathrm{Bcl}-2$ to $\mathrm{Bax}(P<0.05$, Figure $4 \mathrm{~g})$. Flow cytometry and TUNEL assay results consistently indicated that the percentage of cells undergoing apoptosis was significantly lower in the DKK1 siRNA group than in the NC group $(P<0.05$, Figures $4 \mathrm{~h}-\mathrm{k})$. 
a
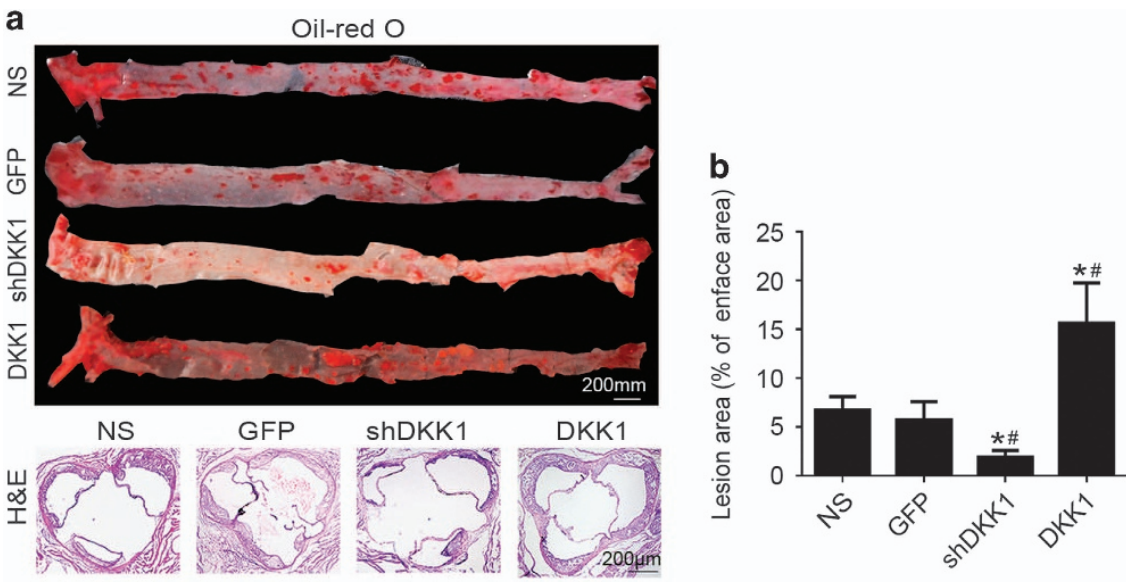

C
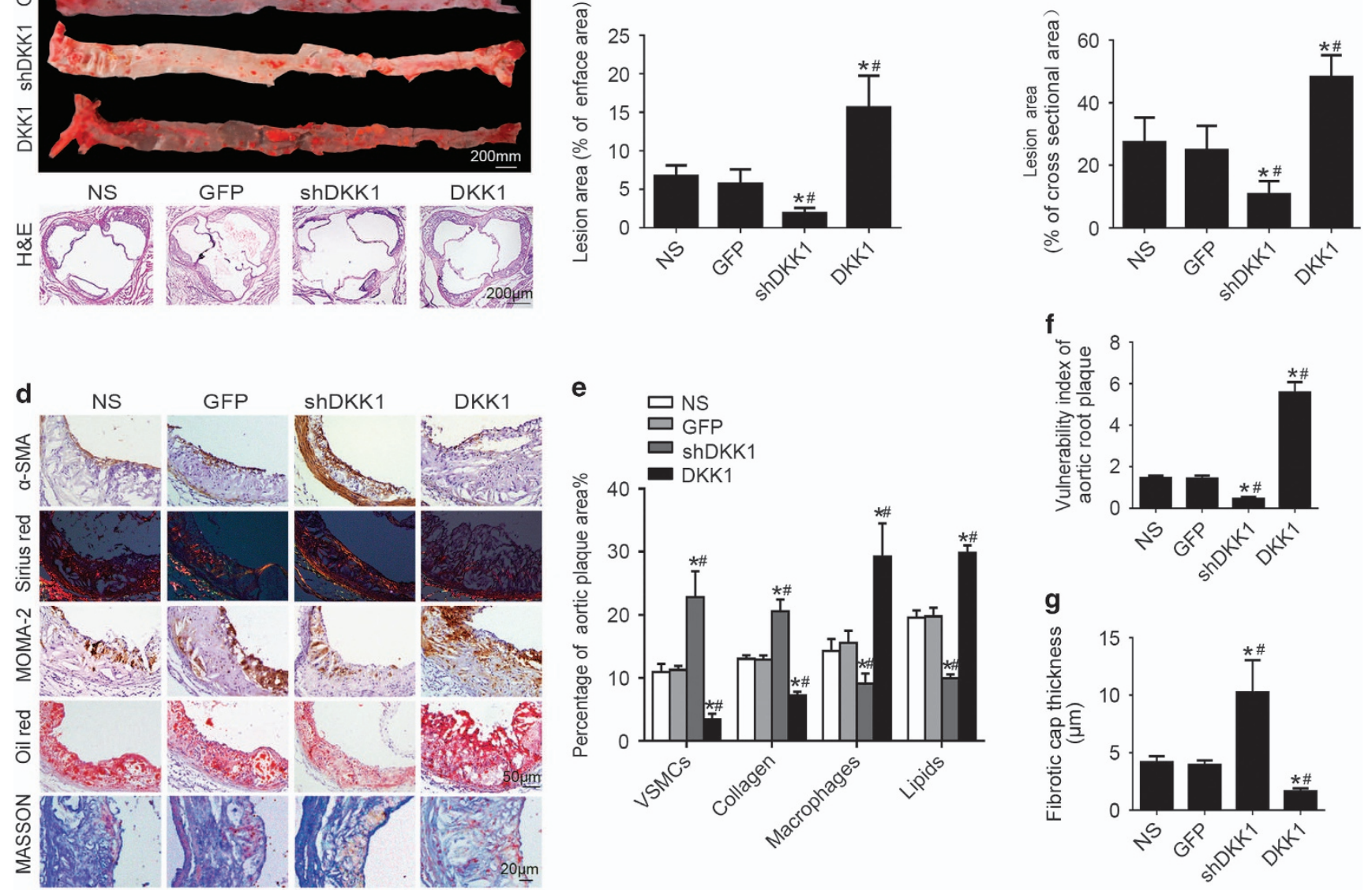

t
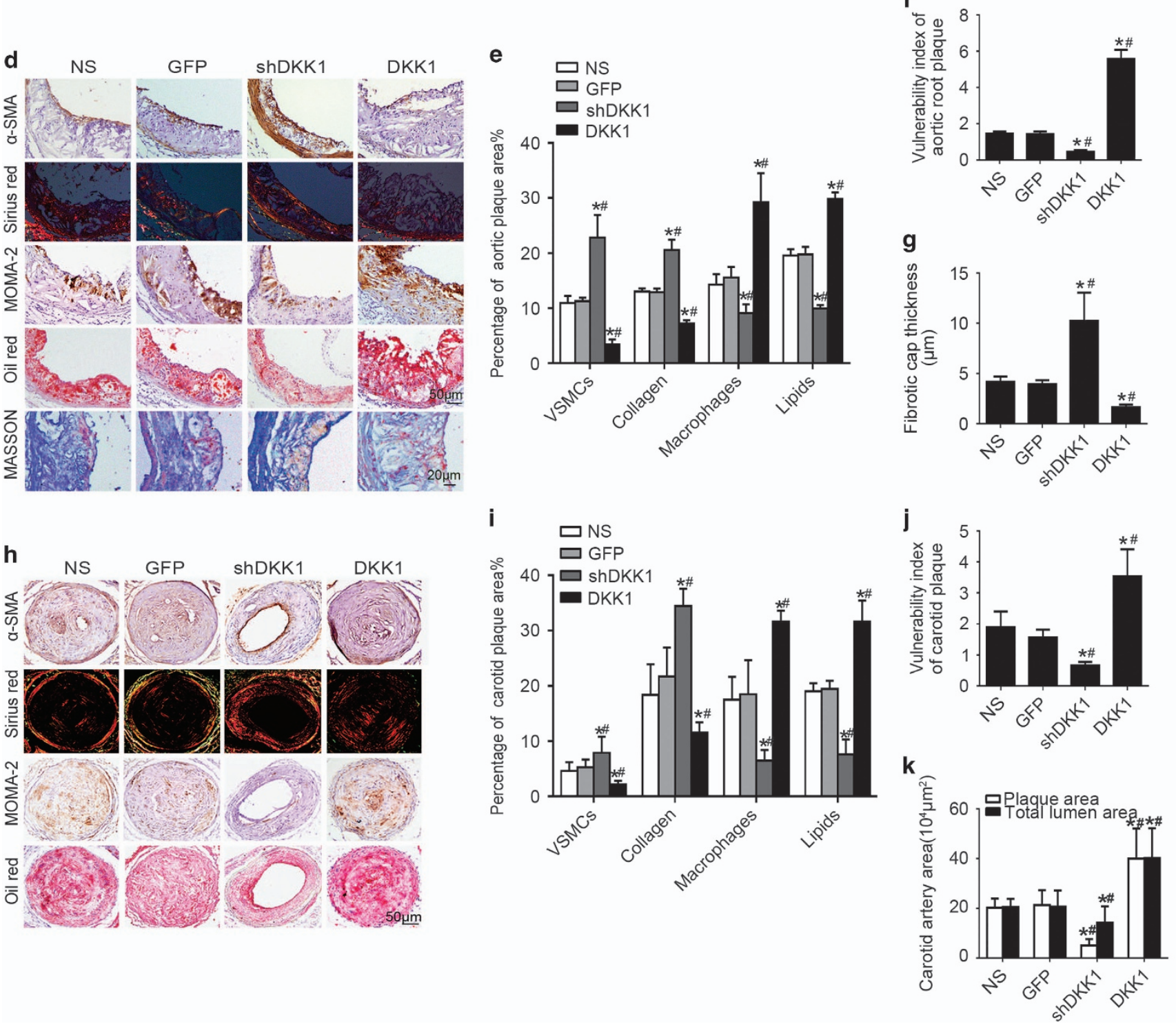

Figure 2 Influence of DKK1 on plaque formation and stability in ApoE - / - mice. (a) En face Oil Red O staining of aortas and cross-sectional aortic root lesions with H\&E staining in four groups of mice (NS, GFP, shDKK1, and DKK1). (b) Quantitative analysis of en face aortic lesions expressed as percentage lesion area relative to total aorta area. $n=6$. (c). Quantitative analysis of cross-sectional plaque areas in aortic roots. $n=6$. (d-e,h-i) Representative immunohistochemical staining and quantification of plaque content in aortic plaques and carotid artery plaques. $n=6$. (f,j) Quantitative analysis of plaque vulnerability indices of the aortic plaques and carotid artery plaques. (g) Quantitative analysis of plaque fibrotic cap thickness of aortic plaques. $n=5$. (k) Quantitative analysis of plaque area and total lumen area in the carotid artery. $n=6$.Data are shown as the mean \pm S.D. ${ }^{*} P<0.05$ versus $N S$; ${ }^{\#} P<0.05$ versus GFP 
a

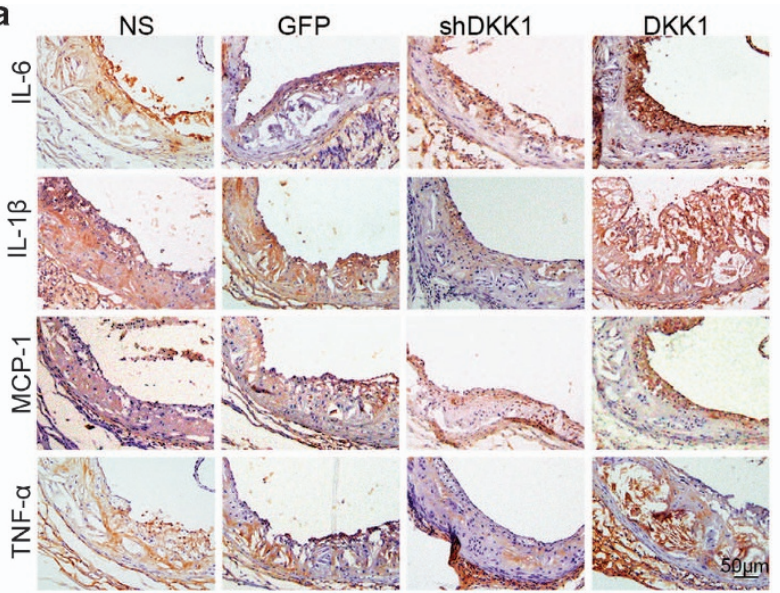

b
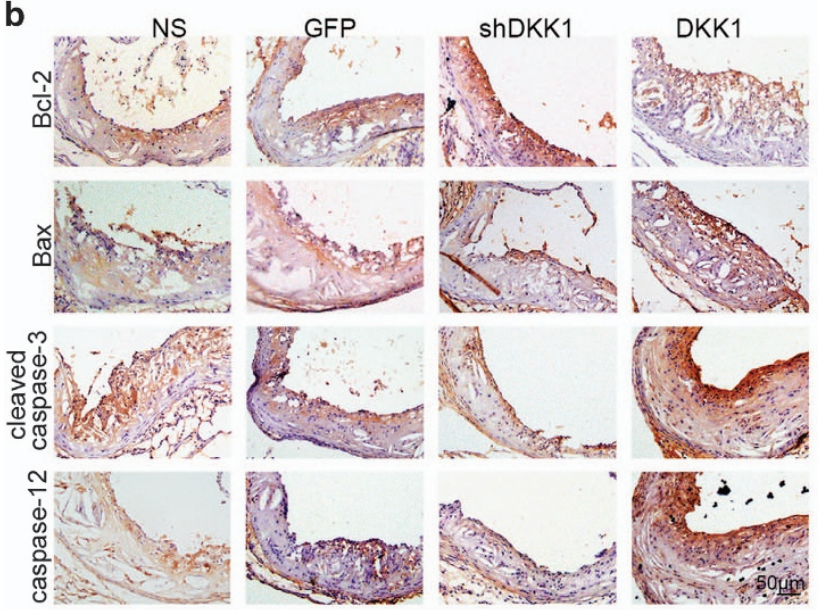

C
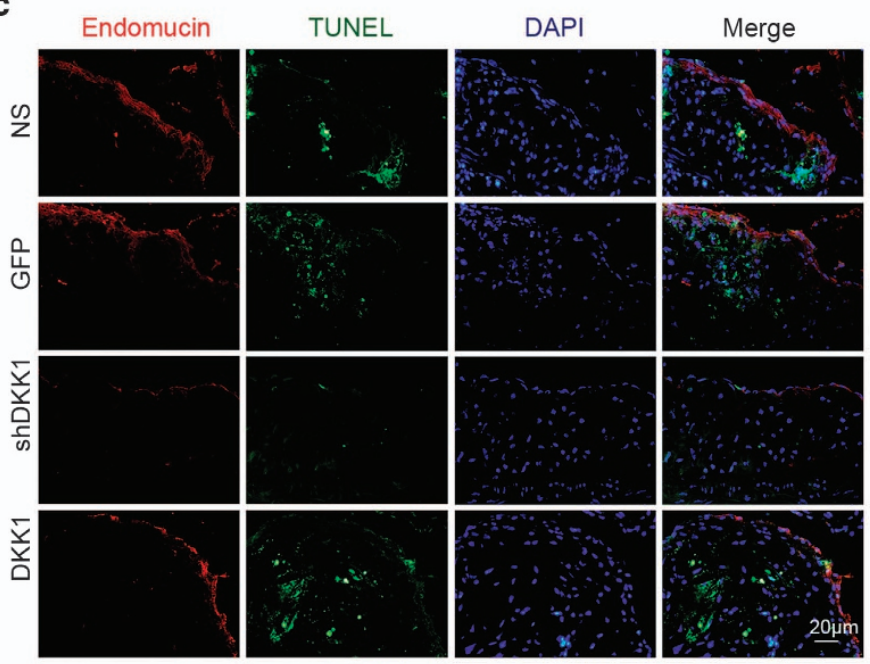

Figure 3 Effects of DKK1 on inflammatory factors and plaque apoptosis in ApoE - / - . (a) Representative immunohistochemical staining and quantification of plaque content of inflammatory factors (IL-6, IL-1 $\beta, M C P-1$, and TNF- $\alpha$ ). $n=6$. (b) Representative immunohistochemical stained images and quantification of Bax, Bcl-2, cleaved caspase-3 and caspase-12 levels in atherosclerotic plaques of ApoE - / - mice. $n=6$. (c) Co-localization and quantitative analysis of TUNEL staining (green) and endomucinpositive areas (red) to measure cellular apoptosis in aortic plaque. DAPI (blue) indicates nuclei. $n=3$. Data are shown as the mean \pm S.D., ${ }^{\star} P<0.05$ versus NS; ${ }^{\#} P<0.05$ versus GFP 
DKK1-induced apoptosis in HUVECs by activating ERS. We next monitored changes in the expression of caspase-12, which is considered to be a marker of ERS-associated apop- tosis in mice. Caspase-12 expression was reduced in the shDKK1 group and enhanced in the DKK1 group $(P<0.05$,

Figure 3a). To further investigate whether DKK1-induced a

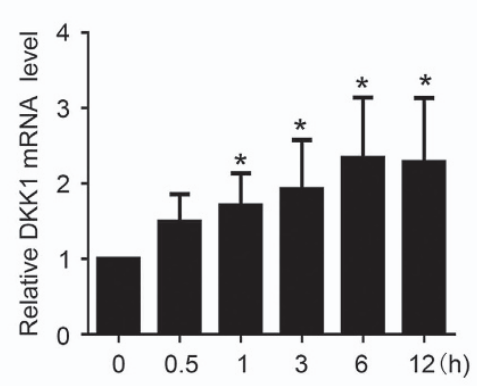

d

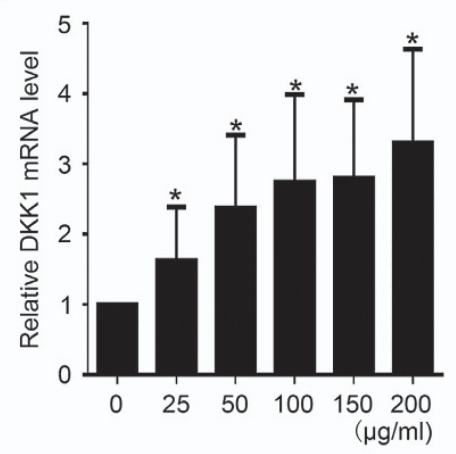

g
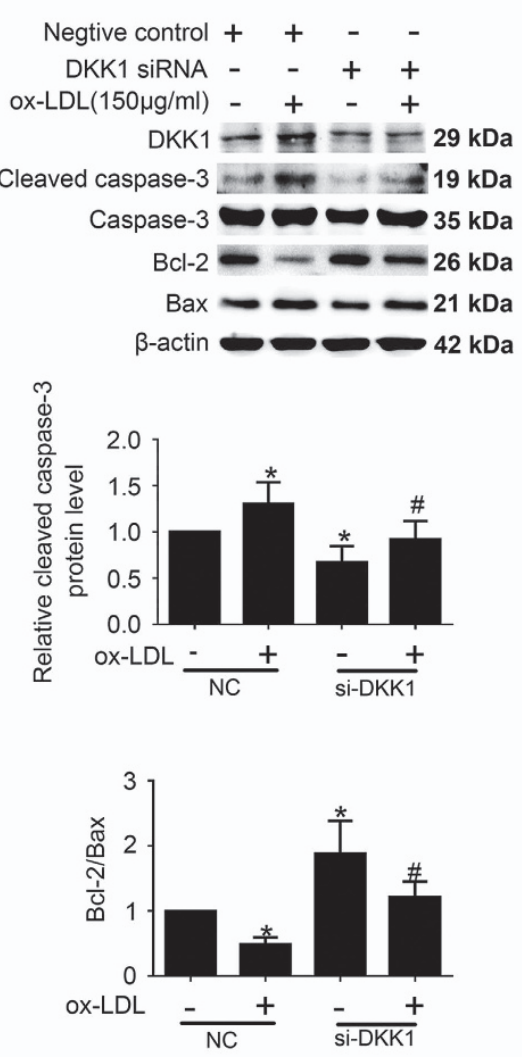

b

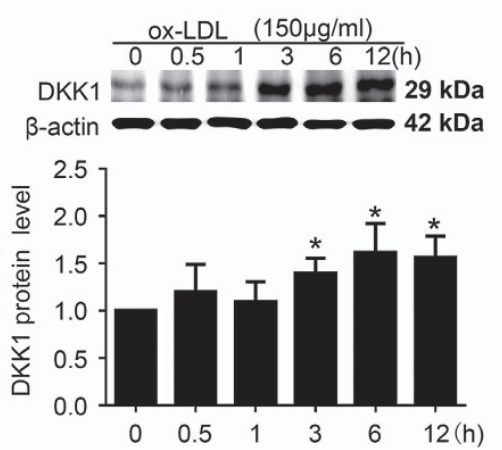

e

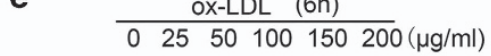

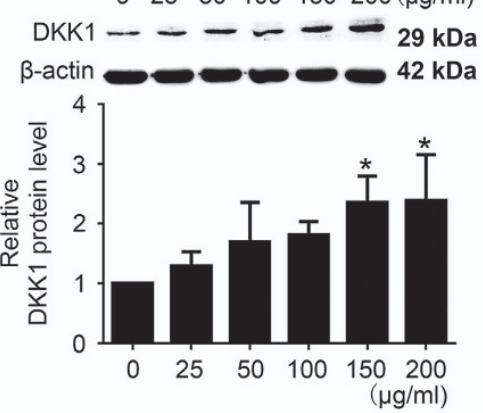

C

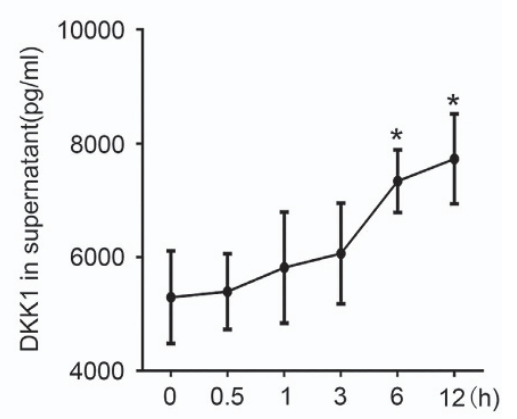

f

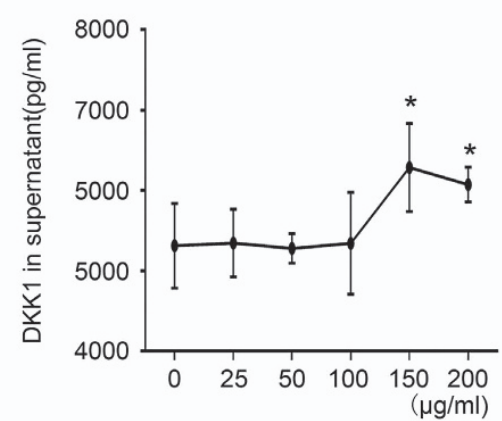

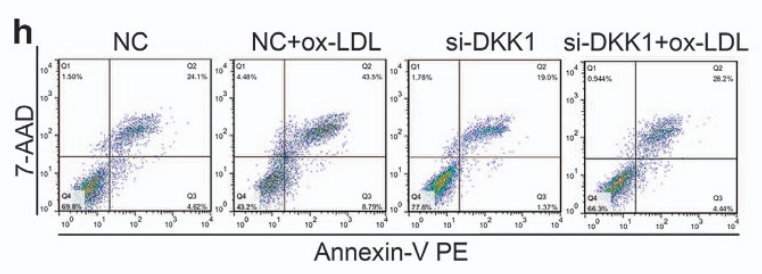
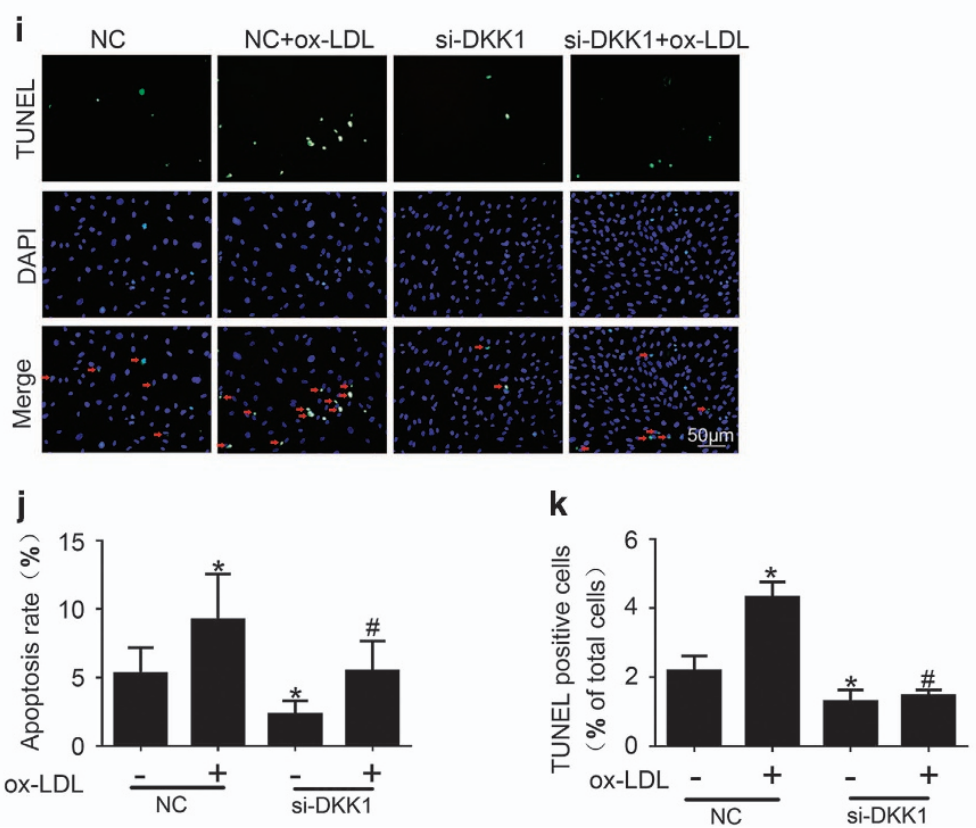
apoptosis through ERS, we treated cells with 4-phenylbutyric acid (4-PBA), an ERS inhibitor. ${ }^{17}$ Treatment with 4-PBA significantly attenuated the increase in cleaved caspase-3 expression and the decrease in the $\mathrm{Bcl}-2 / \mathrm{Bax}$ ratio observed in rDKK1-stimulated HUVECs $(P<0.05$, Figure 5a) or lenti-DKK1-transfected HUVECs $(P<0.05$, Supplementary Figure 3a). Notably, the TUNEL assay and flow cytometry results were consistent with those obtained by Western blotting $(P<0.05$, Figures $5 b-e)$. In summary, we demonstrated that DKK1 induces apoptosis partly via the ERS pathway both in vivo and in vitro.
We also examined the effect of DKK1 on ERS. First, cells transfected with DKK1 siRNA were confirmed to have reduced DKK1 expression relative to cells transfected with NC siRNA. In contrast, cells were treated with ox-LDL or rDKK1 to induce the overexpression of DKK1. To determine whether ox-LDL activates ERS through DKK1, we monitored changes in the levels of eukaryotic initiation factor $2 a$ (eif2a), CHOP, inositol-requiring enzyme 1 (IRE1), sliced X-box-binding protein 1 (XBP1s), transcription factor 6 (ATF6), and GRP78, ${ }^{18}$ which are considered markers of ERS. ${ }^{19}$ All of these markers showed significantly increased protein a
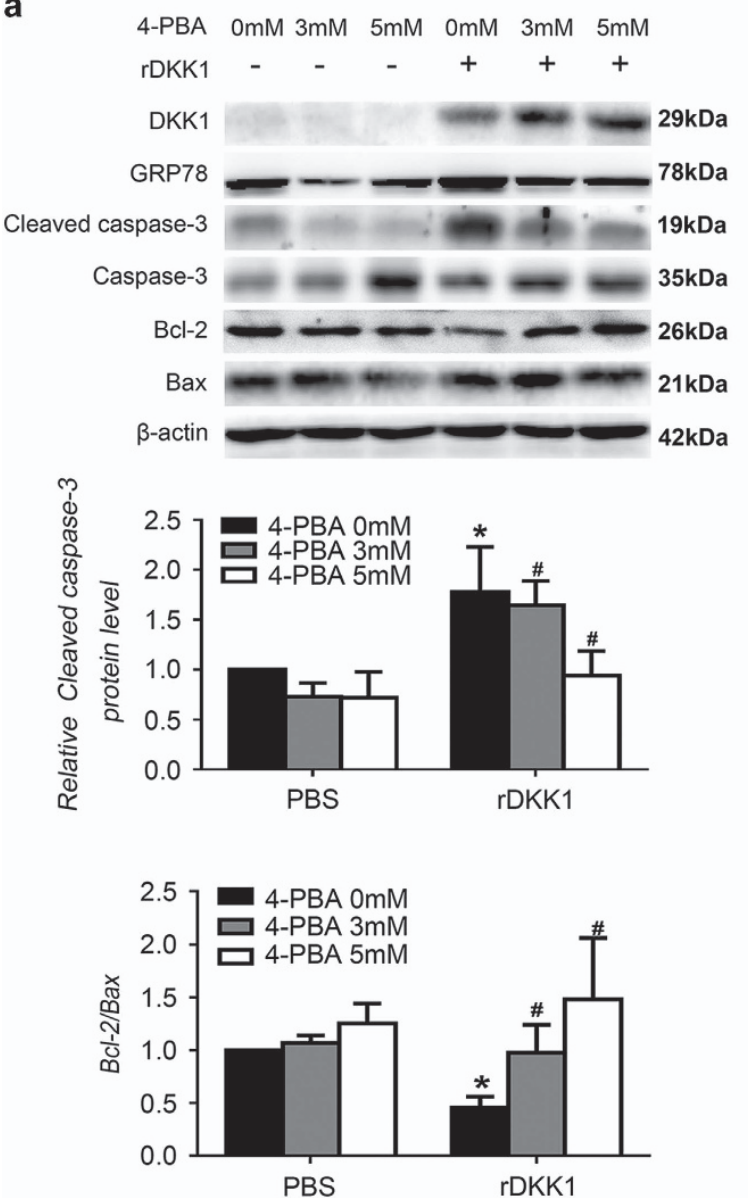

b
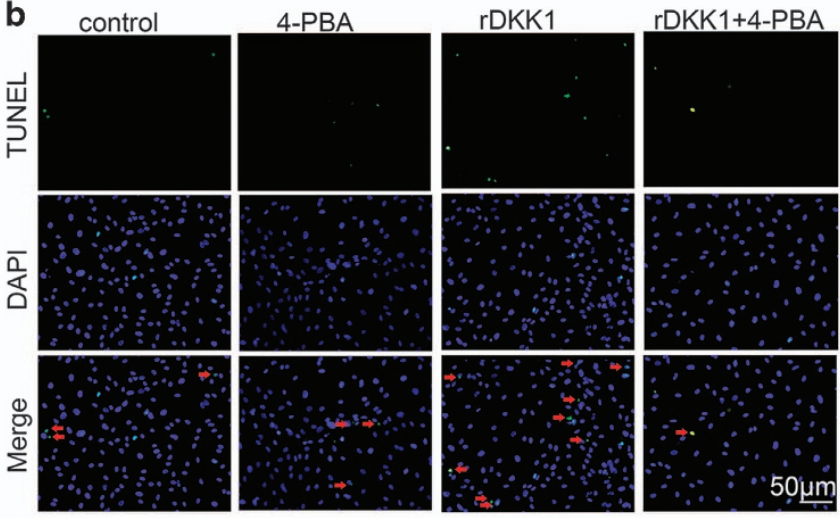

C
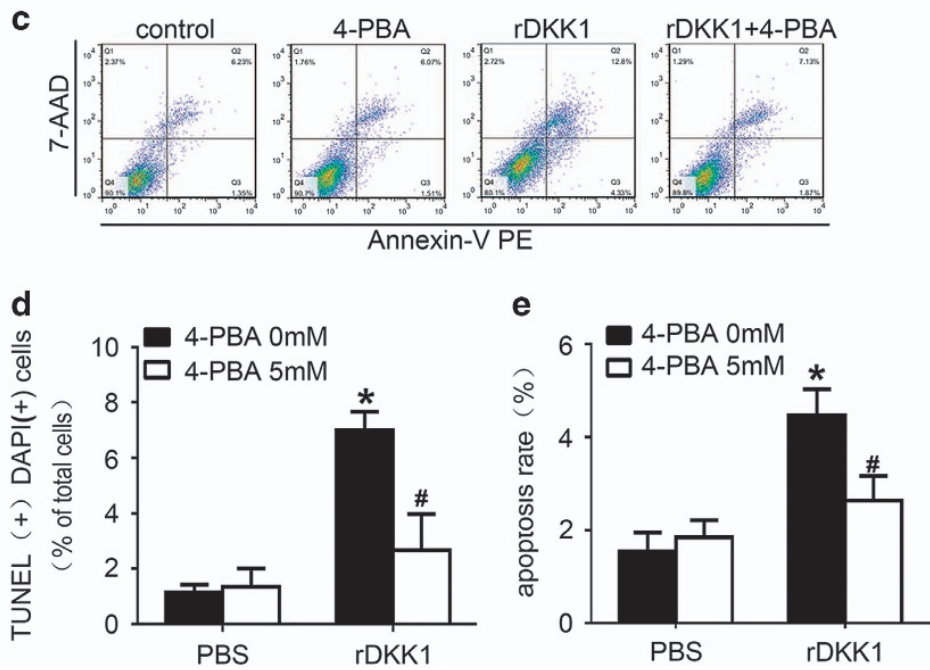

Figure 5 DKK1 promotes apoptosis via endoplasmic reticulum stress in HUVECs. Cells were pretreated with PBS or 4-PBA ( $3 \mathrm{mM}$ or $5 \mathrm{mM}$ ) for $1 \mathrm{~h}$ before and during rDKK1 $(300 \mathrm{ng} / \mathrm{ml})$ treatment. (a) Western blotting to quantify cleaved caspase-3, Bcl-2, and Bax protein expression. $n=6$. (b,d) HUVECs with stained nuclei (green) were considered TUNEL-positive (arrows). The percentage of TUNEL-positive cells was calculated and quantified. $n=3$. (c,e) Flow cytometric analysis for quantification of early apoptotic cells (i.e., Annexin V-positive and 7-AAD-negative cells, lower-right quadrant). $n=3$. Data are shown as the mean \pm S.D. ${ }^{*} P<0.05$ versus control; ${ }^{\#} P<0.05$ versus only rDKK1 treatment

Figure 4 Time- and dose-dependent effects of ox-LDL treatment on the expression of DKK1 and attenuation of ox-LDL-induced apoptosis in HUVECs following DKK1 knockdown. (a-c) Quantification of DKK1 expression in HUVECs treated with ox-LDL (150 $\mu \mathrm{g} / \mathrm{ml})$ for various lengths of time: (a) DKK1 mRNA levels, $n=3$. (b) protein expression levels, $n=6$. (c) Levels in culture supernatant by ELISA, $n=3$. (d-f) Quantification of DKK1 expression in HUVECs treated for $6 \mathrm{~h}$ with various concentrations of ox-LDL: (d) DKK1 mRNA, $n=3$. (e) Protein expression levels, $n=6$. (f) Levels in culture supernatant by ELISA, $n=3$. (g-k) HUVECs were transiently transfected with negative control (NC) and DKK1 siRNA (si-DKK1) for $24 \mathrm{~h}$ and then treated with $150 \mu \mathrm{g} / \mathrm{ml}$ ox-LDL for $6 \mathrm{~h}$. (g) Western blotting to quantify Bax, Bcl-2, cleaved caspase-3 and DKK1 protein levels. $n=6$. (h,j) Flow cytometric analysis to quantify early apoptotic cells (i.e., Annexin V-positive and 7-AAD-negative cells, lower-right quadrant). $n=3$. (i,k) HUVECs with stained nuclei (green) were considered TUNEL-positive (red arrows). The percentage of TUNEL-positive cells was calculated and quantified. $n=6$. Data are shown as the mean \pm S.D. ${ }^{*} P<0.05$ versus the untreated group or $\mathrm{NC} ;{ }^{*} P<0.05$ versus $\mathrm{NC}+\mathrm{Ox}-\mathrm{LDL}$ 
expression in HUVECs following treatment with ox-LDL or rDKK1 $(P<0.05$, Supplementary Figure $4 a)$. In addition, siRNA-mediated silencing of DKK1 reversed ox-LDL-induced ERS $(P<0.05$, Supplementary Figure $4 \mathrm{~b})$. On the basis of these findings, we concluded that DKK1 may induce apoptosis in ox-LDL-treated HUVECs partly via ERS.

DKK1-induced ERS-associated apoptosis through IRE1a and eif2a/CHOP. We have monitored that DKK1 induced the level of eif2a, CHOP, IRE1, XBP1s, ATF6, and GRP78 in HUVECs. To identify the components playing a main role in DKK1-induced, ERS-associated apoptosis, we transfected HUVECs with NC SiRNA, CHOP siRNA, or IRE1a siRNA prior to treatment with $\mathrm{rDKK} 1$ or transfection with lentiDKK $1 .{ }^{20}$ After treatment with rDKK1 or transfection with lentiDKK1, Bax and cleaved caspase-3 protein expression decreased, while $\mathrm{Bcl}-2$ expression increased in cells transfected with CHOP siRNA or IRE1a siRNA compared to those transfected with NC siRNA $(P<0.05$, Figures $6 a$ and $b$, Supplementary Figure $3 \mathrm{~b}-\mathrm{c}$ ). Flow cytometry and TUNEL assay results were consistent with those obtained by Western blotting $(P<0.05$, Figures $6 c-f)$.

To further verify the role of elF2a in DKK1-mediated effects, we also treated cells with salubrinal, which specifically inhibits ERS by preventing elF2a dephosphorylation. ${ }^{21-23}$ Salubrinal significantly attenuated the increased cleaved caspase-3 expression and decreased $\mathrm{Bcl}-2 / \mathrm{Bax}$ expression in rDKK1stimulated HUVECs $(P<0.05$, Figure 7a) and lenti-DKK1 transfected HUVECs $(P<0.05$, Supplementary Figure $3 d)$. Moreover, the flow cytometry and TUNEL assay results were consistent with those obtained by Western blotting $(P<0.05$, Figures $7 \mathrm{~b}-\mathrm{e})$. Generally, DKK1-induced apoptosis via IRE1a and eif2a/CHOP signaling, independent of other ERS transducers.

DKK1-induced ERS-associated apoptosis through activation of the JNK pathway and inhibition of Wnt/ $\beta$-catenin signaling. We transfected cells with DKK1 siRNA to downregulate DKK1 and infected cells with lentivirus to overexpress DKK1. Western blotting revealed that DKK1 activated JNK, while knockdown of DKK1 led to a decrease in JNK phosphorylation $(P<0.05$, Supplementary Fig.5a-5b). To further verify the effect of JNK pathway on ER stress and apoptosis, we pretreated HUVECs with the JNK inhibitor-SP600125 significantly reversed the upward trend in GRP78 and CHOP protein expression observed in HUVECs transfected with lenti-DKK1 $(P<0.05$, Figure 8a). These data indicate that DKK1 induces apoptosis and ERS in HUVECs by targeting and upregulating JNK. Furthermore, Bcl-2 expression increased significantly in lenti-DKK1-transfected HUVECs that were pretreated with SP600125, while Bax expression decreased.

IM-12 activates canonical Wnt signaling, ${ }^{24}$ whereas FH535 acts as an inhibitor of canonical Wnt signaling. ${ }^{25}$ To verify the effect of the canonical Wnt signaling pathway on apoptosis and ERS, we pretreated HUVECs with IM-12 or FH535. HUVECs with pretreated IM-12 significantly reversed the upward trend in GRP78 and IRE1a protein expression in HUVECs transfected with lenti-DKK1 $(P<0.05$, Figure $8 d)$, while HUVECs with pretreated $\mathrm{FH} 535$ reverted the decrease in GRP78 and IRE1a protein expression in HUVECs transfected with DKK1 siRNA $(P<0.05$, Figure $8 e)$. However, the protein levels of JNK and CHOP were not changed in IM-12 or FH535.

\section{Discussion}

This is the first study to describe how DKK1 affects plaque formation and stability in atherosclerosis in ApoE - / - mice, which was accomplished by using lentivirus-mediated silencing and overexpression of the DKK1 gene.The following conclusions were generated: (1) The overexpression of DKK enlarged and destabilized atherosclerotic lesions and increased apoptosis, while inhibition of DKK1 expression hold the formation and vulnerability of atherosclerotic plaques in the whole progression of atherosclerosis; (2) treatment with ox-LDL induces DKK1 expression in HUVECs in a time- and concentration-dependent manner; (3) DKK1 induces ERS through IRE1 $a$ and eif2a/CHOP, leading to apoptosis; and (4) DKK1 activates ERS via both the JNK pathway and canonical Wnt signaling.

Our results show that DKK1 dose not influence the circulating levels of total cholesterol, LDL-C or blood glucose (Supplementary Table 1). Classical pathological studies have demonstrated that plaque components, inflammation factors and apoptosis play important roles in modulating the stability of atherosclerotic plaques. ${ }^{26-28}$ We found that DKK1 silencing reduced macrophage accumulation and increased VSMC numbers in plaques, while DKK1 overexpression augmented plaque vulnerability during the entire process of atherosclerosis.

DKK1 is an antagonist of the Wnt signaling pathway. ${ }^{29-32}$ Ueland et al. ${ }^{9}$ found that DKK1 contributes to the activation of ECs by platelets. In a previous clinical study of patients with ACS, DKK1 plasma levels not only correlated with disease severity but also served as a prognostic predictor of disease, suggesting that DKK1 levels reflect coronary atherosclerosis stability. ${ }^{10}$ In two previous investigations conducted by our group, we found that treatment with ox-LDL promotes DKK1 expression in macrophages, resulting in inhibiting the accumulation of lipids ${ }^{33}$ and that oscillatory shear stress can induce DKK1 expression in ECs through PAR1/CREB. ${ }^{34}$ Both of these biomechanical and haemodynamic factors contribute to the development and destabilization of atherosclerosis. In earlier research, we used a partial carotid ligation model to imitate and induce disturbed flow and acute endothelial injury. ${ }^{34,35}$ Differ from it, we used constrictive silica collars to accelerate atherosclerotic lesion formation. ${ }^{36}$ And we observed the lesion on both right carotid artery and aorta root 12 weeks' atherogenic chow after collar surgery. Thus, in the present study, we demonstrated the negative effects of DKK1 on the formation and instability of atherosclerotic plaques.

In this study, we also observed that the expression of inflammatory factors (e.g., IL-6, IL-1 $\beta$, TNF-a, and MCP-1) decreased in conjunction with the downregulation of DKK1 and increased with upregulation of DKK1. Accumulation of inflammatory factors may induce monocyte recruitment and adhesion to the activated endothelial layer, thereby aggravating plaque instability. ${ }^{6,26-28}$ Several studies have reported that the exogenous inhibition of DKK1 reduced IL-1 $\beta$ and 
a
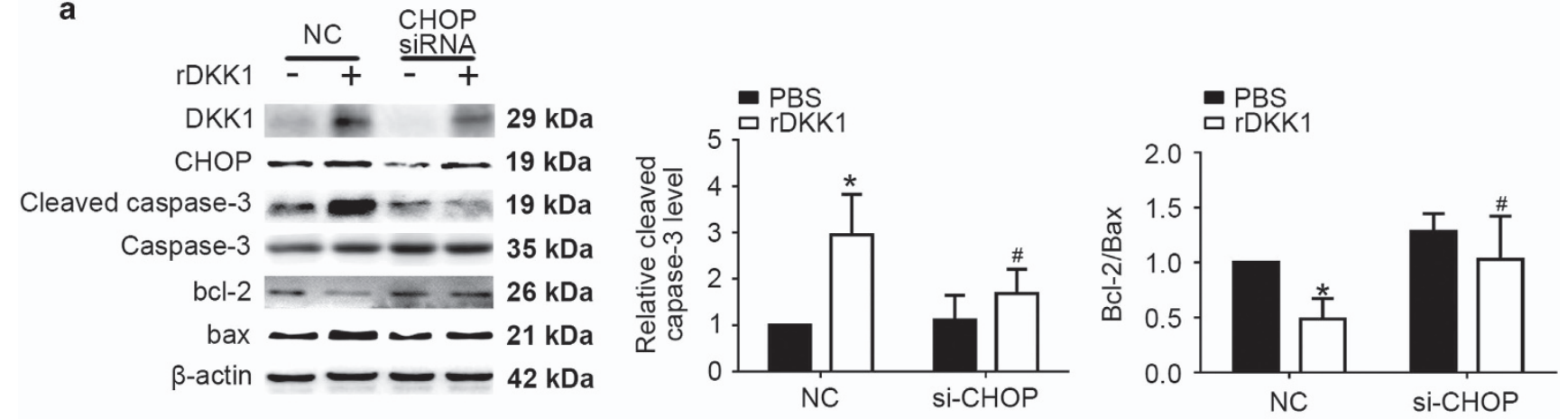

b
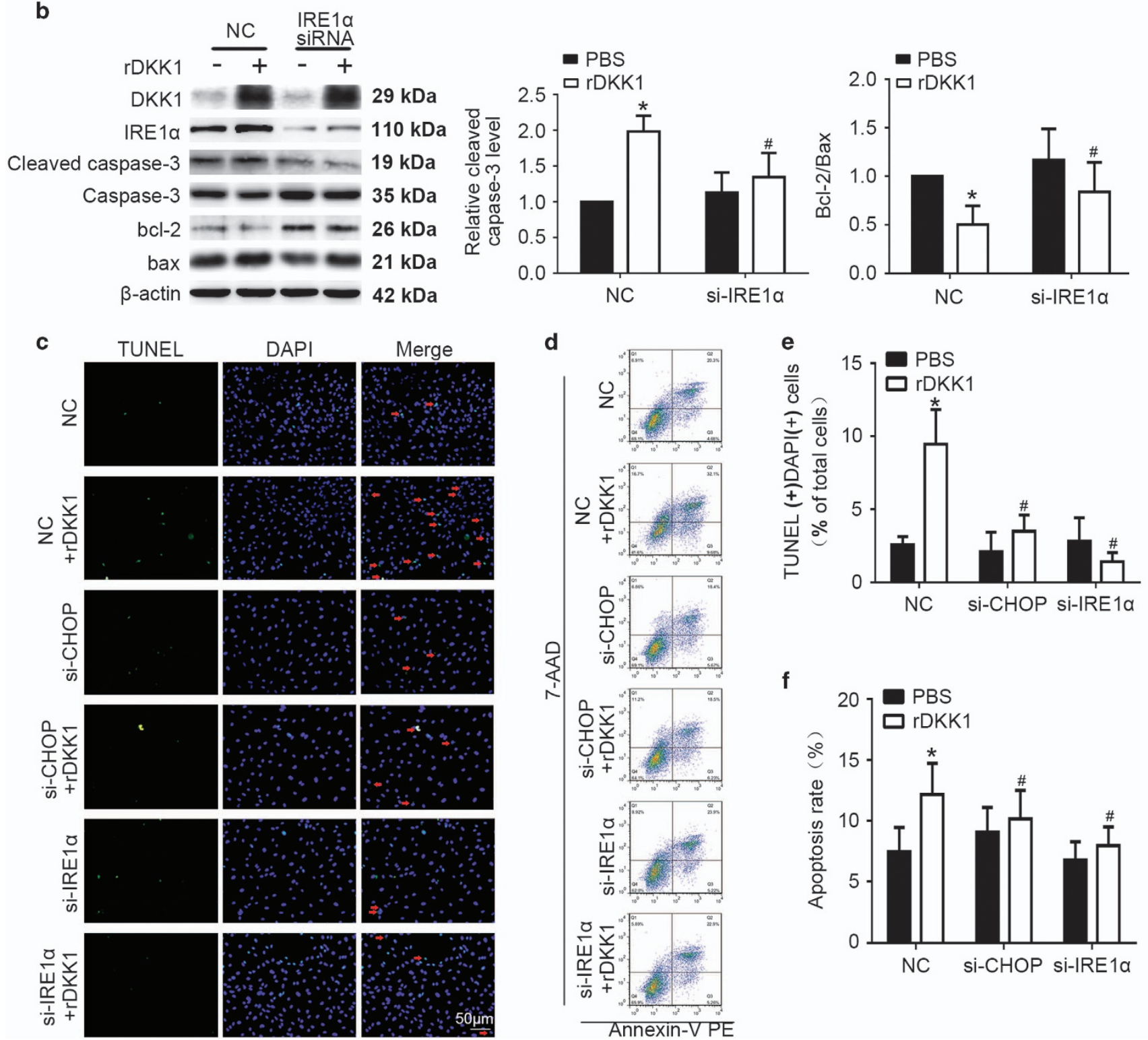

Figure 6 DKK1 activates CHOP and IRE1 $\alpha$ signaling during ERS to induce apoptosis. HUVECs were transiently transfected with negative control (NC) siRNA, CHOP siRNA (si-CHOP) or IRE1 $\alpha$ siRNA (si-IRE1 $\alpha$ ) for $24 \mathrm{~h}$ and then treated with rDKK1 $(300 \mathrm{ng} / \mathrm{ml})$ for $6 \mathrm{~h}$. $(\mathbf{a}, \mathbf{b})$ Western blotting for quantification of the protein levels of cleaved caspase-3, $\mathrm{Bcl}-2$, and Bax. $n=6$. (c,e) HUVECs with stained nuclei (green) were considered TUNEL-positive (arrows). The percentage of TUNEL-positive cells was calculated and quantified. $n=3$. (d,f) Flow cytometric analysis for quantification of early apoptotic cells (i.e., Annexin V-positive and 7-AAD-negative cells, lower-right quadrant). $n=3$. Data are shown as the mean \pm S.D. ${ }^{*} P<0.05$ versus $\mathrm{NC}$ or control; ${ }^{\#} P<0.05$ versus only rDKK1 treatment 
a

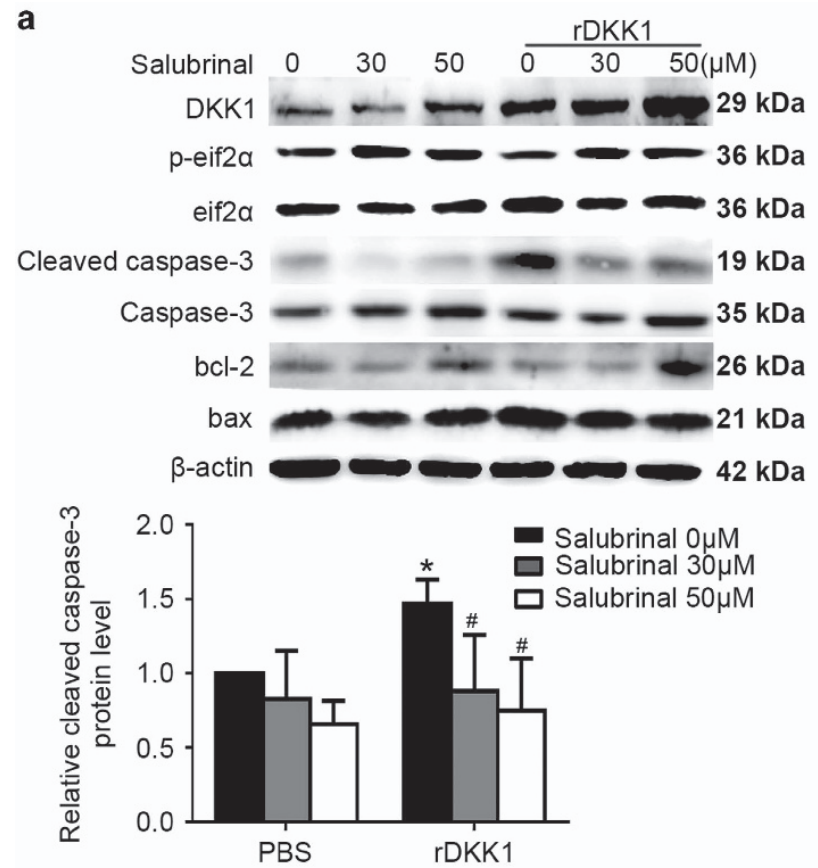

b
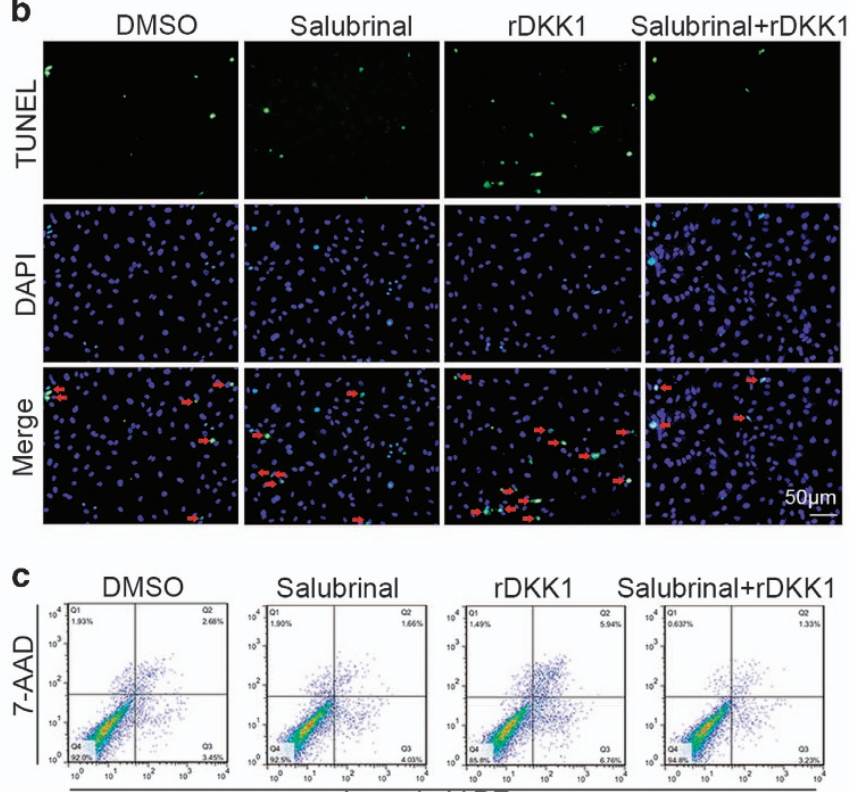

Salubrinal

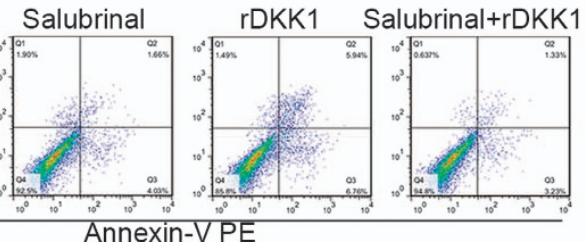

d

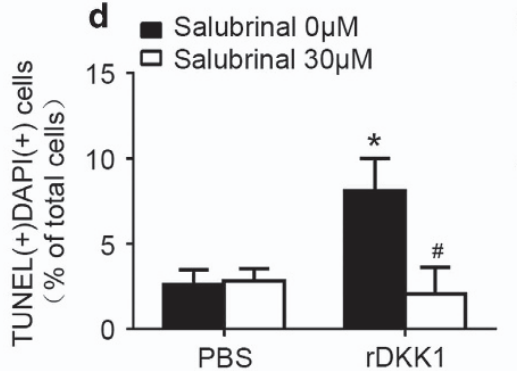

e 15

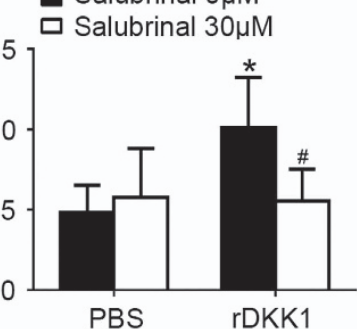

Figure 7 DKK1 activates eif2 $\alpha$ during ERS to induce apoptosis. Cells were pretreated with DMSO or salubrinal $(50 \mu \mathrm{M})$ for $1 \mathrm{~h}$ before rDKK1 (300 ng/ml) treatment. (a) Western blotting for quantification of the protein levels of cleaved caspase-3, Bcl-2, and Bax. $n=6$. (b,d) HUVECs with stained nuclei (green) were considered TUNEL-positive (arrows). The percentage of TUNEL-positive cells was calculated and quantified. $n=3$. (c,e) Flow cytometric analysis for the quantification of early apoptotic cells (i.e., Annexin V-positive and 7-AAD-negative cells, lower-right quadrant). $n=3$. Data are shown as the mean \pm S.D. ${ }^{*} P<0.05$ versus NC or control; ${ }^{\#} P<0.05$ versus only rDKK1 treatment

TNF-a expression, significantly inhibited TNF-a expression in macrophage sand chondrocytes stimulated by lipopolysaccharide, ${ }^{37}$ attenuated angiogenesis, ${ }^{38}$ and decreased monocyte adhesion to HUVECs. ${ }^{39}$ DKK1 has also been shown to affect MMP-3 expression and influence collagen degradation in cartilage. ${ }^{40}$ Furthermore, DKK1 upregulation increases neovascularization. ${ }^{41}$ Thus, DKK1 may lead to plaque instability by augmenting inflammation, adhesion, collagen degradation and neovascularization.

In addition, we found that inhibition of DKK1 expression can attenuate cleaved caspase-3 expression as well as apoptosis. As a major regulator of the Wnt signaling pathway, DKK1 induces cellular apoptosis in many diseases. ${ }^{29-32}$ Overexpression of DKK1 sensitizes cells in brain tumors, ${ }^{42}$ renalcell carcinoma and thyroid cancers ${ }^{43,44}$ to apoptosis. Weng et al. discovered that DKK1 expression is closely correlated with the expression of pro-apoptotic factors (e.g., Bad and caspase-3) in osteoarthritis and inhibition of DKK1 expression reduced caspase- 3 cleavage and alleviated chondrocyte apoptosis by reducing Bax expression and increasing Bcl-2 expression. ${ }^{40,45}$ Cellular apoptosis is ubiquitous in vulnerable plaques; as such, recent studies on vulnerable plaques have mostly focused on inflammation and cellular apoptosis.

Multiple pathophysiological factors, both systemic and localized to the arterial walls, can disturb ER function in ECs, VSMCs, and macrophages during the initiation and progression of atherosclerosis. ${ }^{46}$ Expression of ERS activation markers has been observed in atherosclerotic lesions in humans and animals. ${ }^{47}$ Importantly, ERS-associated apoptosis is correlated with plaque instability and the clinical progression of atherosclerosis. CHOP is only robustly expressed in "vulnerable" plaques that show evidence of lesions and apoptosis. ${ }^{47}$ Moreover, Cominacini et al. revealed that persistent ERS is related to abnormal numbers of apoptotic cells in vulnerable plaques. ${ }^{37}$ Consistent with these findings, our results showed that inhibition of DKK1 expression remarkably reduced the expression of ERS-associated apoptotic markers (e.g., caspase-12) in ApoE-/- mice. Another study found that disrupting the secretion of human Wnt5a, a Wnt pathway agonist, induced ERS in mammalian cells, revealing a correlation between Wnt secretion and 
a

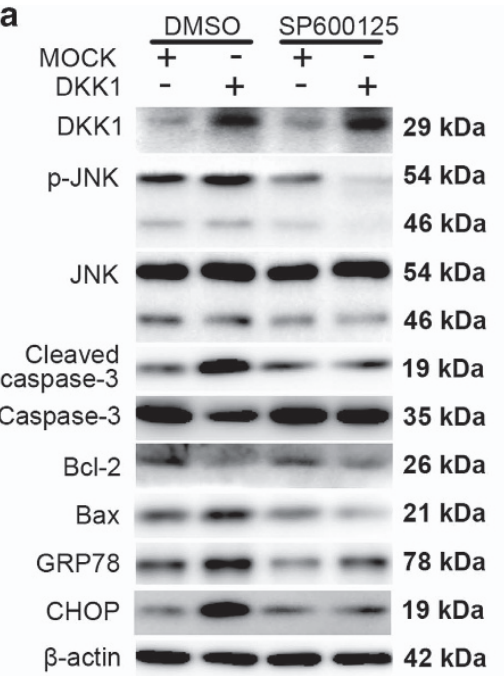

d

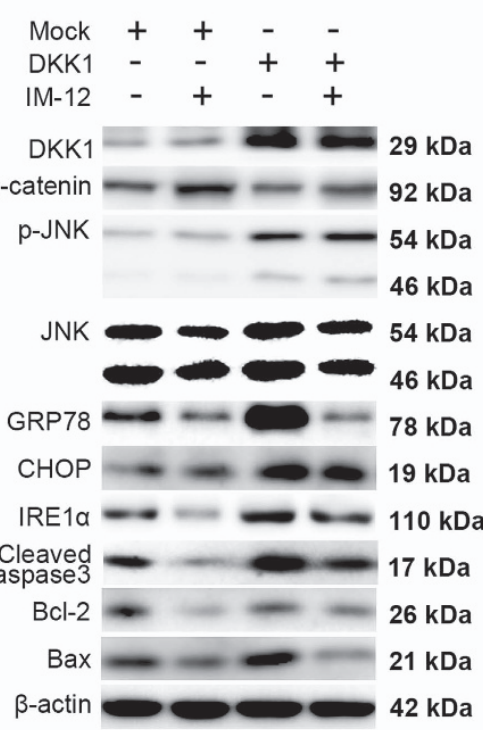

$\begin{array}{ll} & \square \text { DMSO } \\ & \square \text { DKK1+DMSO } \\ \text { b } & \text { SP600125 } \\ \text { DKK1+SP600125 }\end{array}$

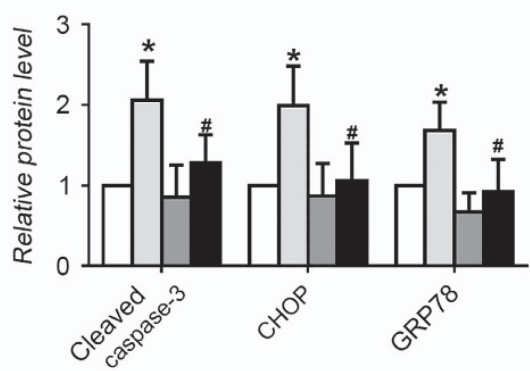

e $\begin{array}{rllll}\text { NC } & + & + & - & - \\ \text { ShDKK1 } & - & - & + & + \\ \text { FH535 } & - & + & - & + \\ \text { DKK1 } & - & - & - & \end{array}$

$\beta$-catenin $\longrightarrow 2 \mathrm{kDa}$ p-JNK - - $-54 \mathrm{kDa}$ $46 \mathrm{kDa}$ JNK $--54 \mathrm{kDa}$

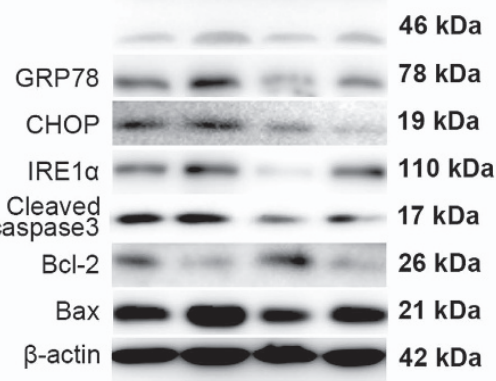

i ㅁMSO

므 D D 535 SO+DKK1 siRNA

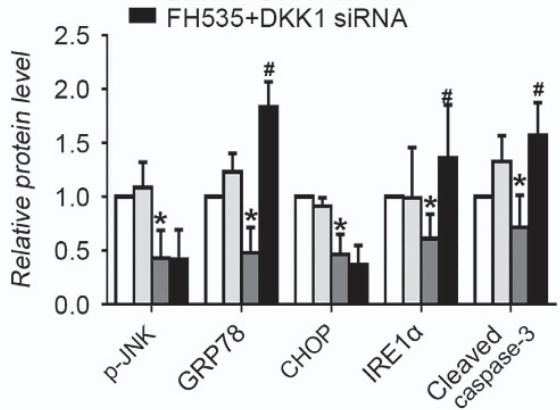

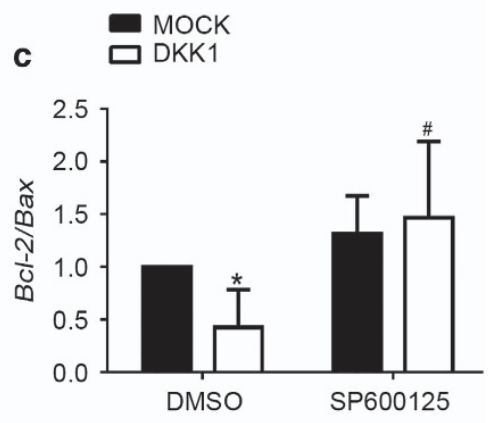

f

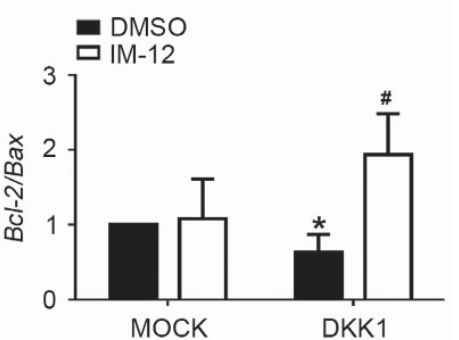

g

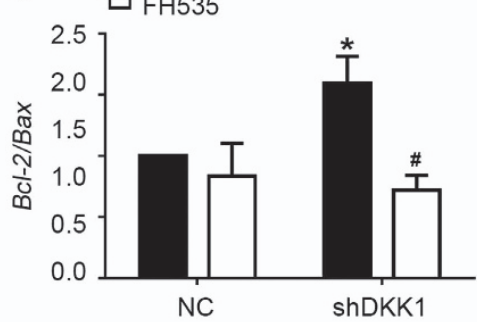

j

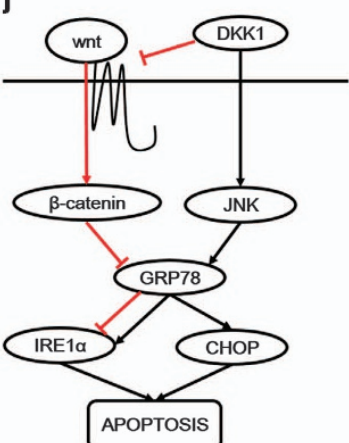

Figure 8 DKK1 induces apoptosis by promoting ERS via activation of the JNK pathway and inhibition of canonical Wnt signaling. (a-c) Cells were pretreated with DMSO or SP600125 $(10 \mu \mathrm{M})$ for $1 \mathrm{~h}$ before transfection with lenti-DKK1. Western blotting for quantification of cleaved caspase-3, Bcl-2, Bax, CHOP and GRP78 protein levels. $n=6$. Data are shown as the mean \pm S.D. ${ }^{*} P<0.05$ versus DMSO; ${ }^{*} P<0.05$ versus rDKK1 treatment only. (d,f, $\left.\mathrm{h}\right)$ Cells were pretreated with DMSO or IM-12 $(3 \mu \mathrm{M})$ for $1 \mathrm{~h}$ before and during transfection with lenti-DKK1. Western blotting for quantification of cleaved caspase-3, Bcl-2, Bax, p-JNK, CHOP, IRE1 $\alpha$, and GRP78 protein levels. $n=6$. Data are shown as the mean \pm S.D. ${ }^{*} P<0.05$ versus DMSO; ${ }^{\#} P<0.05$ versus lenti-DKK1 transfection. (e,g,i) Cells were pretreated with DMSO or FH535 (30 $\left.\mu \mathrm{M}\right)$ for $1 \mathrm{~h}$ before and during transfection with DKK1 siRNA. Western blotting for quantification of cleaved caspase-3, Bcl-2, Bax, p-JNK, CHOP, IRE1 $\alpha$ and GRP78 protein levels. $n=6$. Data are shown as the mean \pm S.D. ${ }^{*} P<0.05$ versus DMSO; ${ }^{\#} P<0.05$ versus DKK1 shRNA transfection. (j) Proposed model of the DKK1 signaling pathway responsible for cell apoptosis 
ERS. ${ }^{13}$ Our results indicate that DKK1 induces ERS and facilitates apoptosis in atherosclerosis.

In our study, ECs were found to be important sources of DKK1, as demonstrated by the co-localization of DKK1 with endomucin, an EC marker, in atherosclerotic lesions in ApoE - / - mice, which was in accord with the previous investigation. ${ }^{9}$ Interestingly, the observed areas of endomucin-positive ECs localization were almost equivalent to those that were stained positive for DKK1. Thus, even though DKK1 is expressed in different cell types, endothelial cells presumably played the most important roles other than smooth muscle cells and macrophages. While many different cells participate in ACS, ${ }^{48}$ apoptosis of ECs is a key event in the initiation of atherosclerotic plaque formation ${ }^{47}$ and the progression to advanced atherosclerosis, which is vulnerable to rupture. ${ }^{3,6,7,49-51}$ We found that both SMCs and macrophages take up the GFP-labeled DKK1 from co-cultured ECs (Supplementary Figure 6a). In previous studies, recombinant DKK1 blocked the proliferation of $\mathrm{VSMCs}^{52}$ and inhibited foam cell formation in macrophages. ${ }^{33}$ Besides, inhibition of DKK1 expression can attenuate the inflammation of macrophages (Supplementary Figure $6 b-c$ ). Thus, the effects of DKK1 in promoting the dysfunction and apoptosis of endothelial cells may be the "starting point" of DKK1's effects on cross-talk with other cells in atherogenesis. ADMA ${ }^{53}$ and $\mathrm{ET}-1^{54}$ are markers of endothelial cell dysfunction. In our study, we also found that ADMA and ET-1 were positively correlated with DKK1 levels in plasma from healthy controls, unstable angina pectoris and acute myocardial infarction patients $(P<0.05$, Supplementary Figure 7$)$. Therefore, we focused on ECs to experimentally determine the function of DKK1 in vitro.

Inhibition of DKK1 expression decreased ox-LDL-induced apoptosis and ERS in HUVECs. Prolonged and unresolvable ERS is known to induce apoptosis. ${ }^{55,56}$ In particular, ERS promotes EC apoptosis via caspase-12 and the mitochondrial pathway. ${ }^{57,58}$ ERS has also been induced in ECs via pathological shear stress, ${ }^{59}$ hypoxia, ${ }^{18,57,58}$ and increased GRP78, CHOP and caspase-12 expression. Here, for the first time, we showed that ox-LDL treatment activates ERS apoptosis via the JNK pathway, thereby promoting HUVECs apoptosis.

Numerous studies have shown that different cell types have specific ERS components; however, the effect of these variations on EC apoptosis is unclear. In 2005, Boyce et al. ${ }^{21}$ reported that salubrinal, a selective inhibitor of eif2a dephosphorylation, protects cells from ERS. In the present study, we used salubrinal, CHOP siRNA and IRE1a siRNA to inhibit various components associated with $E R S$ and to investigate the role of eif2a, CHOP and IRE1a in ERS-associated apoptosis. Ultimately, we found that DKK1-induced ERSassociated apoptosis via IRE1a and eif2a/CHOP signaling. DKK1 is an antagonist of the canonical Wnt signaling pathway. We also found DKK1 can influence GRP78, IRE1a and apoptosis factors through $\mathrm{Wnt} / \beta$-catenin signaling. However, $W n t / \beta$-catenin signaling did not change the protein levels of JNK and CHOP.

In conclusion, inhibition of DKK1 expression effectively decrease plaque stability by attenuating ERS-mediated cellular apoptosis through intiating the JNK pathway and inhibiting Wnt/ßcatenin. Moreover, the IRE1 1 and eif2a/CHOP pathways were found to participate in the activation of ERS. However, the mechanism underlying the augmentations of ERS by DKK1 remains to be determined. Notably, lentiviruses have low specificity in vivo. Therefore, in future experiments we will use EC-specific DKK1 KO mice and macrophagespecific DKK1 KO mice to determine the exact role of DKK1 in atherosclerosis through its action on various cell types. Although our data are preliminary, these findings might lead to new and promising methods for the treatment of atherosclerosis.

\section{Materials and Methods}

Ethics statement. All in vivo protocols involving animal care and experiments complied with the Guide for Care and Use of Laboratory Animals published by the United States National Institutes of Health (NIH Publication, 8th Edition, 2011) and the Animal Management Rules of the Chinese Ministry of Health (Document No. 55, 2001). All the in vivo experiments were approved by the Animal Care Committee of Shandong University. All the in vitro experimental protocols were approved by the Key Laboratory of Cardiovascular Remodeling and Function Research, Qilu hosipital, China. Human plasma samples were obtained from 72 patients of $45-75$ years old patients in Qilu hospital. The research protocol was approved by the ethical committee of Qilu hospital, Shandong University.

Atherosclerosis animal model protocol. A total of $120 \mathrm{ApoE}-/$ - mice (eight- week- old males) were purchased from the Peking University Animal Research Center (Beijing). All mice were fed atherogenic chow (i.e., a high-fat diet with $0.25 \%$ cholesterol and 15\% cocoa butter) (Figure 1a). The atherosclerotic model was created as previously described. ${ }^{33}$ We applied constrictive silica collars to the right carotid artery (RCA) to accelerate atherosclerotic lesion formation and investigated size, components, and vulnerability index in both aortic and carotid plaques. Pentobarbital sodium was used for anesthesia via intraperitoneal injection $(40 \mathrm{mg} / \mathrm{kg}$ ) when placing the constrictive collars. The mice were randomly divided into four groups ( $n=30$ each): a normal saline group (NS), an empty lentivirus group (GFP), a DKK1i lentivirus group (shDKK1), and a DKK1 lentivirus group (DKK1). Eight weeks after the surgery, a $200 \mu \mathrm{l}$ suspension $\left(4^{*} 10^{8}\right.$ TU DKK1i or DKK1 lentivirus per $\mathrm{ml}$ ) was injected into each mouse through the tail vein. The mice were killed 4 weeks post-transfection using pentobarbital sodium $(50 \mathrm{mg} / \mathrm{kg}$, i. p.) before exsanguination by perfusion via the abdominal aorta with PBS.

Cell culture. HUVECs were obtained from ScienCell Research Laboratories (Carlsbad, CA, USA) and cultivated in endothelial cell medium (ECM) (ScienCell, Carlsbad, CA) supplemented with 10\% fetal bovine serum (FBS) and 1\% penicillin/ streptomycin at $37{ }^{\circ} \mathrm{C}$ in $5 \% \mathrm{CO}_{2}$. Cells from passages 4 to 8 were used for experiments. THP-1 cells obtained from the American Type Culture Collection (ATCC) $160 \mathrm{nM}$ phorbol myristate acetate (PMA) was used overnight for THP-1 cell differentiation into macrophages.

Lentiviral silencing and overexpression-vector construction. To generate a lentivirus-mediated silencing vector, the lentivirus vector $p G L V 3 / H 1 / G F P$ +Puro (pGLV3) was purchased from GeneChem (Shanghai, China), and a short-hairpin RNA sequence targeting DKK1 and or scrambled control RNA, was cloned into the vector. The following duplexes targeted murine DKK1: sense 5'-TCACCATCAAGCCAGCAAT-3'; antisense 5'-TCACCATCAAGCCAGCAAT-3'.

To achieve lentivirus-mediated DKK1 overexpression, the lentiviral vector LV5 was purchased from GenePharma Co., Ltd. (Shanghai, China), and the full-length coding sequence of either human or mouse DKK1 C-terminally tagged with green fluorescent protein (GFP) was cloned into the vector. A vector cloned with GFP alone was used as a negative control (NC).

SiRNA and RNA interference. Upon reaching 40-60\% confluence, HUVECs were transfected with specific siRNA or negative control siRNA (GenePharma, Shanghai, China) (shown in Supplementary Table 1) using Lipofectamine 3000 (Thermo Fisher Scientific, Waltham, MA, USA) in Opti-MEM (Gibco, Thermo Fisher Scientific, Waltham, MA, USA). After $6 \mathrm{~h}$ of transfection, the medium was replaced with complete ECM, and the cells were cultured for an additional $24 \mathrm{~h}$. The transfected cells were treated with ox-LDL, recombinant DKK1 
(rDKK1) or lentivirus DKK1 at the designated concentrations and for the indicated times.

Biochemical measurements. The mice were fasted overnight. Blood samples were collected and centrifuged. Serum levels of total cholesterol, triglycerides, low-density lipoprotein cholesterol (LDL-C), high-density lipoprotein cholesterol and blood sugar were measured via enzymatic assay using an automatic biochemical analyser (Roche Cobas Integra 800, Basel, Switzerland).

Histopathology and immunohistochemistry. The whole aorta, the aortic root and the RCA were dissected, removed, fixed in $4 \%$ formaldehyde overnight at $4{ }^{\circ} \mathrm{C}$, embedded in OCT compound, prepared into 5 - $\mu \mathrm{m}$-thick sections. The cryosections were stained with haematoxylin and eosin for plaque morphology, oil red $\mathrm{O}$ for lipids, picrosirius red for collagen and Masson's trichrome staining for fibrous cap. After blocking in $5 \%$ bovine serum albumin (BSA) in PBS, the sections were incubated with primary antibodies (shown in Supplementary Table 2) overnight at $4^{\circ} \mathrm{C}$ and then with an HRP Detection System (ZSGB-BIO, Beijing, China). Detection was subsequently performed using DAB ( $3,3^{\prime}$-diaminobenzidine) (ZSGB$B I O)$. Plaques stained with picrosirius red were viewed under polarized light. The areas of collagen, VSMCs, extracellular lipid deposits and macrophages were recorded as the percentage of positive area divided by the plaque area in 20 highpower fields $\left(20^{*}\right)$. The vulnerability index was calculated using the following formula: (macrophage staining\%+lipid staining\%)/(VSMC staining\%+collagen staining\%). ${ }^{60}$ Staining in the plaque was quantified using Image-Pro Plus 6.0 software (Media Cybernetics, Rockville, MD, USA) and a colour CCD video microscope (OLYMPUS, Tokyo, Japan).

Immunofluorescence staining and microscopy. The cryosections were blocked with $1 \%$ BSA and incubated with primary antibodies (shown in Supplementary Table 2) at $4{ }^{\circ} \mathrm{C}$ overnight. The sections were washed with PBS, and incubated with FITC- or TRITC-conjugated secondary antibodies. Nuclei were stained with 4', 6-diamidino-2-phenylindole (DAPI; 1:2000, Roche, Mannheim, Germany) for $5 \mathrm{~min}$. The samples were rinsed three times in PBS, and were examined under an epifluorescence microscope, and data were analyzed using Image-Pro Plus 6.0 software (Media Cybernetics).

Aortic ring experiments in vitro. Thirty $A p o E-/-$ mice were injected NS, DKK1i or DKK1 lentivirus respectively. The mice were killed 2 weeks after transfection. The thoracic aortic rings $2-3 \mathrm{~mm}$ in depth that included the entire endothelium were cut and mounted in a $620 \mathrm{M}$ Multi myograph system (DMT, Aarhus, Denmark) containing $10 \mathrm{ml}$ of Krebs bicarbonate solution maintained at $37^{\circ} \mathrm{C}$. After standardization, norepinephrine $\left(10^{-5} \mathrm{M}\right)$ was added to the bath to precontract the aortic ring, and stabilize for $5 \mathrm{~min}$. Ach $\left(10^{-7}, 10^{-6}, 10^{-5} \mathrm{M}\right)$ was successively added to the chamber to test for endothelial relaxation. The relaxation responses were calculated as a percentage of the norepinephrine pre-contraction. All the data were collected using Powerlab software (AD Instruments, Bella Vista, NSW, Australia).

Western blot analysis. HUVECs and tissue samples were lysed using RIPA buffer containing $1 \mathrm{mM}$ phenylmethylsulfonyl fluoride (Invitrogen, Carlsbad, CA, USA) and collected by centrifugation at $14000 \times \mathrm{rpm}$ for $10 \mathrm{~min}$. Equal amounts of proteins and pre-stained protein ladder (Thermo Fisher Scientific) were separated on $10 \%$ SDS-PAGE gels, transferred to methanol-activated polyvinylidene fluoride membranes with a $0.45 \mu \mathrm{m}$ pore size (Millipore, Billerica, MA, USA), and incubated with primary antibodies overnight at $4^{\circ} \mathrm{C}$. The membranes were incubated with secondary antibodies (ProteinTech, Rosemont, Penn., USA) the next day for $1 \mathrm{~h}$ $20 \mathrm{~min}$ at room temperature. Bands were visualized using Immobilon ECL substrate (Millipore, Billerica, MA, USA), and blots were imaged with an LAS-4000 luminescent image analyser (Fujifilm USA, Valhalla, NY, USA). Protein expression was quantified using Adobe Photoshop CS6 (Adobe Systems, San Jose, CA, USA), normalized to the $\beta$-actin expression in each sample, and expressed as a percentage of the control. The primary antibodies used in the experiments are shown in Supplementary Table 2.

RNA extraction and quantitative real-time PCR. Total RNA was extracted from HUVECs using TRIzol reagent (Ambion, Life Technologies, Waltham, MA, USA), and it was reverse-transcribed into cDNA using a PrimeScript RT Reagent Kit (TakaRa Biotechnology, Dalian, China). The cDNA (1 ng) was subjected to Q-PCR using SYBR Green (TakaRa Biotechnology) for the relative quantification of mRNA expression. Quantification was accomplished using the 2- $\Delta \Delta \mathrm{Ct}$ method. $\beta$-actin was used to normalize mRNA levels. The reverse-transcription primer sequences used for the target genes are shown in Supplementary Table 2.

ELISAs Kit. The level of DKK1 in mice plasma were determined using a mouse DKK1 ELISA Kit (R\&D, Minneapolis, MN, USA). The level of DKK1 in cell supernatant and human plasma was assessed using a human DKK1 ELISA Kit (R\&D). Other protein levels were determined using the following ELISA kits: ADMA ELISA Kit (BlueGene Biotech, Shanghai, China), ET-1 ELISA Kit (BlueGene Biotech). All kits were used according to the manufacturer's protocol. The analysis was completed at a wave-length of $450 \mathrm{~nm}$ (with reference of $570 \mathrm{~nm}$ ) in an ELISA plate reader.

Flow cytometry. Cell apoptosis was analyzed using an Annexin $\mathrm{V} P E / 7$ Amino-Actinomycin (7-AAD) Apoptosis Detection Kit (BD Pharmingen, San Diego, CA, USA) according to the manufacturer's protocol. The following groups were used to set up compensation and to define quadrants: unstained control cells, cells stained with PE Annexin V (no 7-AAD), and cells stained with 7-AAD (no PE Annexin V). Apoptotic cells were examined using a flow cytometer (BectonDickinson, USA) within $1 \mathrm{~h}$, and the percentage of early apoptotic cells (upper-right quadrant) was measured using FlowJo software (Tree Star, Ashland, OR, USA).

In situ detection of apoptotic cells. Apoptotic cells and apoptotic ECs in aortic root cryosections were determined using an In Situ Apoptosis Fluorescein Detection Kit (Millipore, Billerica, MA, USA).

Statistical analysis. Data were analyzed using SPSS v16.0 (SPSS Inc., Chicago, IL, USA). Data are presented as the mean \pm S.D. of at least three independent experiments. Normality of variables distribution was tested by the Kolmogorov-Smirnov test. Comparisons were analyzed using Student's $t$-test or one-way ANOVA followed by Bonferroni post hoc test. $P<0.05$ was considered statistically significant.

\section{Conflict of Interest}

The authors declare no conflict of interest.

Acknowledgements. This study was supported by grants from the National Natural Science Foundation of China (No. 81270404 and 81470559 ).

1. Ross R. Atherosclerosis-an inflammatory disease. N Engl J Med 1999; 340: 115-126.

2. Pober JS, Sessa WC. Evolving functions of endothelial cells in inflammation. Nat Rev Immunol 2007; 7: 803-815.

3. Otsuka F, Finn AV, Yazdani SK, Nakano M, Kolodgie FD, Virmani R. The importance of the endothelium in atherothrombosis and coronary stenting. Nat Rev Cardiol 2012; 9: 439-453.

4. Gimbrone MA Jr., Garcia-Cardena G. Endothelial Cell Dysfunction and the Pathobiology of Atherosclerosis. Circ Res 2016; 118: 620-636.

5. Sumpio BE, Riley JT, Dardik A. Cells in focus: endothelial cell. Int J Biochem Cell Biol 2002; 34: $1508-1512$.

6. Deanfield JE, Halcox JP, Rabelink TJ. Endothelial function and dysfunction: testing and clinical relevance. Circulation 2007; 115: 1285-1295.

7. Ehara S, Ueda M, Naruko T, Haze K, Itoh A, Otsuka M et al. Elevated levels of oxidized low density lipoprotein show a positive relationship with the severity of acute coronary syndromes. Circulation 2001; 103: 1955-1960.

8. Niehrs C. Function and biological roles of the Dickkopf family of Wnt modulators. Oncogene 2006; 25: 7469-7481.

9. Ueland T, Otterdal K, Lekva T, Halvorsen B, Gabrielsen A, Sandberg WJ et al. Dickkopf-1 enhances inflammatory interaction between platelets and endothelial cells and shows increased expression in atherosclerosis. Arterioscler Thromb Vasc Biol 2009; 29 : 1228-1234.

10. Wang L, Hu XB, Zhang W, Wu LD, Liu YS, Hu B et al. Dickkopf-1 as a novel predictor is associated with risk stratification by GRACE risk scores for predictive value in patients with acute coronary syndrome: a retrospective research. PLOS ONE 2013; 8: e54731.

11. Mahua M, Svetlana S, Concepcion RE, Lan C, Tohru T, Lauren G et al. Dickkopf1 Is Required for Embryonic Head Induction and Limb Morphogenesis in the Mouse. Dev Cell 2001; 1: 423-434.

12. Myoishi M, Hao H, Minamino T, Watanabe K, Nishihira K, Hatakeyama K et al. Increased endoplasmic reticulum stress in atherosclerotic plaques associated with acute coronary syndrome. Circulation 2007; 116: 1226-1233.

13. Zhang $P$, Zhou L, Pei C, Lin X, Yuan Z. Dysfunction of Wntless triggers the retrograde Golgi-to-ER transport of wingless and induces ER stress. Sci Rep 2016; 6: 19418. 
14. Christopher AJ, Nyall RL, Haoyu C, Kye WP, Dominique S, Rebecca AS et al. Robo4 stabilizes the vascular network by inhibiting pathologic angiogenesis and endothelial hyperpermeability. Nat Med 2008; 14: 448-453.

15. Hongyou Y, Corey SM, Alexandra FT, Lynn W, Robert K, Catherine MR et al. Angiopoietin-2 attenuates angiotensin II-induced aortic aneurysm and atherosclerosis in apolipoprotein E-deficient mice. Sci Rep 2016; 6: 35190.

16. Di Pietro N, Pandolfi A. Physiology and pathophysiology of oxLDL uptake by vascular wall cells in atherosclerosis. Vasc Pharmacol 2016; 84: 1-7.

17. Li X, Xu C, Yang P. c-Jun NH2-terminal kinase 12 and endoplasmic reticulum stress as interdependent and reciprocal causation in diabetic embryopathy. Diabetes 2013; 62 : 599-608.

18. Santos CXC, Nabeebaccus AA, Shah AM, Camargo LL, Filho SV, Lopes LR. Endoplasmic reticulum stress and Nox-mediated reactive oxygen species signaling in the peripheral vasculature: potential role in hypertension. Antioxid Redox Signal 2014; 20: 121-134.

19. Minamino T, Komuro I, Kitakaze M. Endoplasmic reticulum stress as a therapeutic target in cardiovascular disease. Circ Res 2010; 107: 1071-1082.

20. Matsuoka M, Komoike Y. Experimental Evidence Shows Salubrinal, an elF2 $\alpha$ Dephosphorylation Inhibitor, Reduces Xenotoxicant-Induced Cellular Damage. Int J Mol Sci 2015 16: $16275-16287$.

21. Boyce M, Bryant KF, Jousse C, Long K, Harding HP, Scheuner D et al. A selective inhibitor of elF2alpha dephosphorylation protects cells from ER stress. Science 2005; 307 : 935-939.

22. Rao RV, Niazi K, Mollahan P, Mao X, Crippen D, Poksay KS et al. Coupling endoplasmic reticulum stress to the cell-death program: a novel hsp90-independent role for the small chaperone protein p23. Cell Death Differ 2006; 13: 415-425.

23. Hotamisligil GS. Endoplasmic reticulum stress and the inflammatory basis of metabolic disease. Cell 2010; 140: 900-917.

24. Cai T, Sun D, Duan Y, Wen P, Dai C, Yang J et al. WNT/beta-catenin signaling promotes VSMCs to osteogenic transdifferentiation and calcification through directly modulating Runx2 gene expression. Exp Cell Res 2016; 345: 206-217.

25. Kushwaha $P$, Khedgikar V, Gautam J, Dixit $P$, Chillara R, Verma $A$ et al. A novel therapeutic approach with Caviunin-based isoflavonoid that en routes bone marrow cells to bone formation via BMP2/Wnt-beta-catenin signaling. Cell Death Dis 2014; 5: e1422.

26. Naghavi M, Libby P, Falk E, Casscells SW, Litovsky S, Rumberger J et al. From vulnerable plaque to vulnerable patient: a call for new definitions and risk assessment strategies: part I. Circulation 2003; 108: 1664-1672.

27. Virmani R, Burke AP, Farb A, Kolodgie FD. Pathology of the vulnerable plaque. J Am Coll Cardiol 2006; 47: C13-C18.

28. Falk E, Nakano M, Bentzon JF, Finn AV, Virmani R. Update on acute coronary syndromes: the pathologists' view. Eur Heart J 2013; 34: 719-728.

29. Wirths O, Waha A, Weggen S, Schirmacher P, Kühne T, Goodyer CG et al. Overexpression of human Dickkopf-1, an antagonist of wingless/WNT signaling, in human hepatoblastomas and Wilms' tumors. Lab Invest 2003; 83: 429-434.

30. Pinzone JJ, Hall BM, Thudi NK, Vonau M, Qiang YW, Rosol TJ et al. The role of Dickkopf-1 in bone development, homeostasis, and disease. Blood 2009; 113: 517-525.

31. Caraci F, Busceti C, Biagioni F, Aronica E, Mastroiacovo F, Cappuccio I et al. The Wnt antagonist, Dickkopf-1, as a target for the treatment of neurodegenerative disorders. Neurochem Res 2008; 33: 2401-2406.

32. Gustafson B, Smith U. The WNT inhibitor Dickkopf 1 and bone morphogenetic protein 4 rescue adipogenesis in hypertrophic obesity in humans. Diabetes 2012; 61 : 1217-1224.

33. Zhang Y, Ge C, Wang L, Liu X, Chen Y, Li M et al. Induction of DKK1 by ox-LDL negatively regulates intracellular lipid accumulation in macrophages. FEBS Lett 2015; 589: 52-58.

34. Li M, Liu X, Zhang Y, Di M, Wang H, Wang L et al. Upregulation of Dickkopf1 by oscillatory shear stress acceleratesatherogenesis. J Mol Med (Berl) 2016; 94: 431-441.

35. Douglas N, Chih-Wen N, Amir R, Jin S, Klaudia B, Alexander L et al. Partial carotid ligation is a model of acutely induced disturbed flow, leading to rapid endothelial dysfunction and atherosclerosis. Heart Circ Physiol 2009; 297: H1535-H1543.

36. von der Thüsen $\mathrm{JH}$, van Berkel TJ, Biessen EA. Induction of rapid atherogenesis by perivascular carotid collar placement in apolipoprotein E-deficient and low-density lipoprotein receptor-deficient mice. Circulation 2001; 103: 1164-1170.

37. Cominacini L, Garbin U, Mozzini C, Stranieri C, Pasini A, Solani E et al. The atherosclerotic plaque vulnerability: focus on the oxidative and endoplasmic reticulum stress in orchestrating the macrophage apoptosis in the formation of the necrotic core. Curr Med Chem 2015; 22 : 1565-1572.

38. Smadja DM, D'Audigier C, Weiswald LB, Badoual C, Dangles-Marie V, Mauge L et al. The Wnt antagonist Dickkopf-1 increases endothelial progenitor cell angiogenic potential. Arterioscler Thromb Vasc Biol 2010; 30: 2544-2552.
39. Verma S, Buchanan MR, Anderson TJ. Endothelial function testing as a biomarker of vascular disease. Circulation 2003; 108: 2054-2059.

40. Weng LH, Wang CJ, Ko JY, Sun YC, Wang FS. Control of Dkk-1 ameliorates chondrocyte apoptosis, cartilage destruction, and subchondral bone deterioration in osteoarthritic knees. Arthritis Rheum 2010; 62: 1393-1402.

41. Aicher A, Kollet O, Heeschen C, Liebner S, Urbich C, Ihling C et al. The Wnt antagonist Dickkopf-1 mobilizes vasculogenic progenitor cells via activation of the bone marrow endosteal stem cell niche. Circ Res 2008; 103: 796-803.

42. Liu G, Su L, Hao X, Zhong N, Zhong D, Singhal S et al. Salermide up-regulates death receptor 5 expression through the ATF4-ATF3-CHOP axis and leads to apoptosis in human cancer cells. J Cell Mol Med 2012; 16: 1618-1628.

43. Cho SW, Lee EJ, Kim H, Kim SH, Ahn HY, Kim YA et al. Park YJ.Dickkopf-1 inhibits thyroid cancer cell survival and migration through regulation of $\beta$-catenin/E-cadherin signaling. Mol Cell Endocrinol 2013; 366: 90-98.

44. Hirata H, Hinoda Y, Nakajima K, Kawamoto K, Kikuno N, Ueno K et al. Wnt antagonist DKK1 acts as a tumor suppressor gene that induces apoptosis and inhibits proliferation in human renal cell carcinoma. Int J Cancer 2011; 128: 1793-1803.

45. Weng LH, Wang CJ, Ko JY, Sun YC, Su YS, Wang FS. Inflammation induction of Dickkopf-1 mediates chondrocyte apoptosis in osteoarthritic joint. Osteoarthritis Cartilage 2009; 17 : 933-943.

46. Alex XZ, Ira T. The UPR in atherosclerosis. Semin Immunopathol 2013; 35: 321-332.

47. Nathanson D, Nystrom T. Hypoglycemic pharmacological treatment of type 2 diabetes: targeting the endothelium. Mol Cell Endocrinol 2009; 297: 112-126.

48. Hansson GK. Inflammation, atherosclerosis, and coronary artery disease. N Engl J Med 2005; 352: 1685-1695.

49. Lusis AJ. Atherosclerosis. Nature 2000; 407: 233-241.

50. Libby P, Ridker PM, Hansson GK. Progress and challenges in translating the biology of atherosclerosis. Nature 2011; 473: 317-325.

51. Liu M, Xiang G, Lu J, Xiang L, Dong J, Mei W. TRAIL protects against endothelium injury in diabetes via Akt-eNOS signaling. Atherosclerosis 2014; 237: 718-724.

52. Zhuang Y, Mao JQ, Yu M, Dong LY, Fan YL, Lv ZQ et al. Hyperlipidemia induces vascular smooth muscle cell proliferation involving Wnt/beta-catenin signaling. Cell Biol Int 2016; 40: $121-130$.

53. Chen S, Li N, Deb-Chatterji M, Dong Q, Kielstein JT, Weissenborn K et al. Asymmetric dimethyarginine as marker and mediator in ischemic stroke. Int J Mol Sci 2012; 13: 15983-16004.

54. Jansson PA. Endothelial dysfunction in insulin resistance and type 2 diabetes. $J$ Intern Med 2007; 262: 173-183.

55. Lazzari C, Peggion C, Stella R, Massimino ML, Lim D, Bertoli A et al. Cellular prion protein is implicated in the regulation of local ca2+ movements in cerebellar granule neurons. J Neurochem 2011; 116: 881-890.

56. Oakes SA, Papa FR. The role of endoplasmic reticulum stress in human pathology. Ann Rev Pathol 2015; 10: 173-194.

57. Scull CM, Tabas I. Mechanisms of ER stress-induced apoptosis in atherosclerosis. Arterioscler Thromb Vasc Biol 2011; 31: 2792-2797.

58. Gorman AM, Healy SJ, Jager R, Samali A. Stress management at the ER: regulators of ER stress-induced apoptosis. Pharmacol Ther 2012; 134: 306-316.

59. Haas MJ, Kurban W, Shah H, Onstead-Haas L, Mooradian AD. Beta blockers suppress dextrose-induced endoplasmic reticulum stress, oxidative stress, and apoptosis in human coronary artery endothelial cells. Am J Ther 2016; 23: e1524-e1531.

60. Shiomi M, Ito T, HirouchiY, Enomoto M. Fibromuscular cap composition is important for the stability of established atherosclerotic plaques in mature WHHL rabbits treated with statins. Atherosclerosis 2001; 157: 75-84.

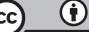

Cell Death and Disease is an open-access journal published by Nature Publishing Group. This work is licensed under a Creative Commons Attribution 4.0 International License. The images or other third party material in this article are included in the article's Creative Commons license, unless indicated otherwise in the credit line; if the material is not included under the Creative Commons license, users will need to obtain permission from the license holder to reproduce the material. To view a copy of this license, visit http://creativecommons.org/licenses/by/4.0/

(C) The Author(s) 2017 